\title{
Does exercise improve sleep for adults with Insomnia? \\ A Systematic Review with Quality Appraisal.
}

\author{
Author A: Hayley Lowe a
}

Authors B-G: Gillian Haddock a b, Lee D. Mulligan c, Lynsey Gregg a , Lesley-Anne Carter d, Annabel Fuzellier-Hart ${ }^{a}$, Simon D. Kyle ${ }^{*}$

${ }^{a}$ University of Manchester, Division of Psychology and Mental Health, School of Health Sciences, Zochonis Building, Second Floor, Brunswick Street, Manchester, England, UK. M13 9PL.

${ }^{b}$ Greater Manchester Mental Health NHS Foundation Trust, Trust Headquarters, Bury New Road, Prestwich, Manchester, England, UK. M25 3BL.

c Greater Manchester Mental Health NHS Foundation Trust, Ramsgate House Community Mental Health Team, Ramsgate Street, Lower Broughton, Salford, England, UK. M7 2YL.

d University of Manchester, Centre for Biostatistics, J ean McFarlane Building, Oxford Road, Manchester, England, UK. M13 9PL.

e University of Oxford, Sleep and Circadian Neuroscience Institute, Nuffield Department of Clinical Neurosciences, Level 6, West Wing, John Radcliffe Hospital, Oxford, England, UK. OX3 9DU.

${ }^{*}$ Corresponding Author: University of Oxford, Sleep and Circadian Neuroscience Institute, Nuffield Department of Clinical Neurosciences, Level 6, West Wing, John Radcliffe Hospital, Oxford, England, UK. OX3 9DU.

Telephone: +44 (0) 1865 618675. Email address: simon.kyle@ndcn.ox.ac.uk. 


\section{Summary}

Insomnia is recognised as the most prevalent sleep disorder. Untreated insomnia carries a heavy burden for patients and society. Exercise is proposed as a safe, inexpensive, and accessible non-pharmacological treatment. To the author's knowledge this is the first systematic review to investigate the sleep-enhancing effects of exercise by focusing exclusively on controlled trials comprising poor sleepers only and examining interventions consistent with national guidelines. Using a narrative synthesis, this review aimed to identify whether exercise improves objective and subjective sleep outcomes for people with insomnia.

Five papers including participants with insomnia disorder, and six papers including participants with insomnia symptoms were identified through electronic database searches (CINAHL plus, Psycl NFO, EMBASE, MEDLINE, SPORTDiscus, CENTRAL) and quality assessed using the Clinical Trial Assessment Measure.

We found that exercise interventions led to improvements in subjective sleep quality for people with insomnia disorder and insomnia symptoms. However, exercise only improved objective and subjective measures of sleep continuity (sleep onset latency and sleep efficiency) for people presenting with insomnia symptoms, with a reduction in sleep onset latency being the most consistently observed effect across studies.

The reliability of significant findings is reduced by methodological limitations. Recommendations are made to improve the quality of future research.

Key words: exercise, physical activity, insomnia, sleep, systematic review 


\begin{tabular}{|ll|}
\hline \multicolumn{2}{|l|}{ Abbreviations } \\
& \\
AIS & Athens Insomnia Scale \\
BNSQ & Basic Nordic Sleep Questionnaire \\
CBT & Cognitive behavioural therapy \\
CTAM & Clinical Trials Assessment Measure \\
HAE & high-intensity aerobic exercise \\
ISI & Insomnia Severity Index \\
ITT & intention to treat analysis \\
MAE & moderate-intensity aerobic exercise \\
MRE & moderate-intensity resistance exercise \\
NRS & non-randomised controlled study \\
OA & older adult \\
PSD & Pittsburgh Sleep Diary \\
PSQI & Pittsburgh Sleep Quality Index \\
PSG & polysomnography \\
QOL & quality of life \\
RCT & randomised controlled trial \\
SE & sleep efficiency \\
SMD & standardised mean difference \\
SOL & sleep onset latency \\
SQ & sleep quality \\
SQAW & Sleep Questionnaire and Assessment of Wakefulness \\
TST & total sleep time \\
UK & United Kingdom \\
US & United States \\
WASO & wake-time after sleep onset \\
WHO & World Health Organisation \\
& \\
\hline
\end{tabular}




\section{Introduction}

Insomnia: Nature, Prevalence and Impact

Insomnia, a serious form of sleep disturbance, constitutes a considerable public health problem (Colten $\&$ Altevogt, 2006). It is recognised as the most prevalent sleep disorder (Morin \& Benca, 2012), and one of the most common complaints in primary care services (Hohagen et al., 1993; Simon \& VonKorff, 1997; Aikens \& Rouse, 2005). Insomnia can present either as a symptom or a disorder (Harvey, 2001), either independently or comorbidly with substance use, another sleep disorder, psychiatric or medical disorder. Insomnia symptoms include difficulties with the initiation or maintenance of sleep, which occur despite adequate opportunity and circumstances to sleep (American Psychiatric Association, 2013; World Health Organization [WHO], 1992; American Academy of Sleep Medicine, 2005). Importantly, insomnia disorder is diagnosed only when the complaint is chronic and persistent (>3 months), and the sleep disturbance causes clinically significant distress and impairs activities of daily living.

It is estimated that one third of the general population experience insomnia symptoms; $9-15 \%$ have the additional experience of daytime impairment; while $6 \%$ meet formal criteria for insomnia disorder (Ohayon, 2002). Although insomnia symptoms are highly prevalent in the general population, people presenting with symptoms only (i.e. meeting sleep disturbance threshold, without the chronicity and daytime impairment criterion) are not defined as clinical samples.

Untreated insomnia carries a heavy burden for people as evidenced by impairments in daytime functioning, quality of life (QOL) and work productivity (Kyle, Morgan, \& Espie, 2010; Bolge, Doan, Kannan, \& Baran, 2009). It also constitutes a risk factor for new onset physical and mental ill-health (Sivertsen et al., 2014), substance abuse and relapse (Taylor, Lichstein, \& Durrence, 2003), accidents and mortality (Summala \& Mikkola, 1994; Kripke, Garfinkel, Wingard, Klauber, \& Marler, 2002). 
Moreover, insomnia carries a high socioeconomic burden with enhanced healthcare utilization worldwide (Daley, Morin, LeBlanc, Gregoire, \& Savard, 2009).

\section{Current Treatment Approaches}

Despite its ubiquity and associated negative outcomes, insomnia often goes undiagnosed and untreated (Morin, Belleville, Bélanger, \& Ivers, 2011), and there is currently no long-term treatment available in services. The National Institute for Health and Care Excellence (NICE, 2004) in the UK recommends short-term use of hypnotics, but only after non-pharmacological measures have been considered. Despite these recommendations, pharmacotherapy remains the most common treatment offered (Tariq $\&$ Pulisetty, 2008), which may be due to limited knowledge about sleep disorders amongst health professionals and limited access to non-pharmacological treatments (Stores \& Crawford, 1998; Stores \& Wiggs, 1998).

In view of potential risks concerning hazardous side-effects, tolerance, dependency and withdrawal, broader dissemination of evidence-based nonpharmacological therapies is needed to address high rates of sleep disturbance. Indeed, there are various non-pharmacological treatment options available, including behavioural and cognitive approaches. Multicomponent cognitive behavioural therapy for insomnia (CBT-I) is currently considered to be the treatment of first choice for persistent insomnia (van Straten, van der Zweerde, Kleiboer, Cuijpers, Morin \& Lancee, 2017). The aim of this intervention is to examine and change unhelpful thoughts, beliefs and attitudes about insomnia, in addition to any unhelpful behaviours through the added combination of behavioural interventions (e.g. stimulus control, sleep restriction, or relaxation training).

A recent meta-analysis which included 87 randomised controlled trials (RCT's), comparing 118 treatments (3724 patients) to non-treated controls ( 2579 patients), demonstrated that CBT-I, either its components or the full package, was effective in the treatment of insomnia (van Straten et al, 2017). The following effects were observed: 
Insomnia Severity Index (ISI) $(g=0.98)$, Sleep Efficiency (SE) $(g=0.71)$, Pittsburgh Sleep Quality Index (PSQI) ( $g=0.65)$, Wake-time After Sleep Onset (WASO) $(g=0.63)$, Sleep Onset Latency $(\mathrm{SOL})(\mathrm{g}=0.57)$ and Sleep Quality $(\mathrm{SQ})(\mathrm{g}=0.40)$. The smallest effect was for Total Sleep Time (TST) $(g=0.16)$.

While brief or digital forms of CBT-I may help to improve access to insomnia treatment, psychological therapies remain unavailable in clinical practice. Potential reasons include poor understanding amongst Health Care Professionals, but also practical considerations which include financial cost and availability of resources (i.e. necessary delivery by highly trained and specialised clinicians), in addition to patient motivation and compliance (Benca, 2005).

\section{Exercise}

Exercise has been proposed as an alternative treatment for insomnia. There are a number of factors which make this approach attractive. In contrast to sleep medications which carry a number of risk factors, exercise is recognised as a safe, inexpensive, and easily accessible means of improving sleep (Passos, Poyares, Santana, Tufik, \& de Mello, 2012; Youngstedt, O'Connor, \& Dishman, 1997; Driver \& Taylor, 2000; Youngstedt, 2005; Buman \& King, 2010). Unlike traditional psychological interventions such as CBT-I, exercise removes the necessity for a trained and highly specialised clinician to implement the treatment. Exercise can be completed individually or as part of a group, supervised or not, in any location, and at a time which is convenient to the individual. Exercise potentially represents a practical, cost-effective and scalable treatment which is easy to apply and implement in community settings. Exercise can_also be individualised to make it attractive and rewarding to the participant. It can be implemented using wide-ranging and relatively inexpensive or priceless resources (e.g. health centre, gym park, fitness class, media sources), and it appears to fit with a culture becoming increasingly focused upon health and fitness. Beyond effects on sleep, exercise has been shown to confer a variety of other health benefits. This includes, but is not limited to, improved physical 
and mental health (U.S. Department of Health and Human Service, 2008; Penedo \& Dahn, 2005) and reduced risk of mortality (Paffenbarger et al., 1993; Kaplan et al., 2017).

Physical exercise is endorsed by the National Sleep foundation (n.d.) and commonly features in sleep hygiene recommendations (Espie, 2006; Irish, Kline, Gunn, Buysse, \& Hall, 2015). Futhermore, the WHO (2010) currently recommends that 150 minutes of moderate to vigorous intensity (aerobic or resistance) exercise per week is needed to deliver health benefits, which can be accumulated through engaging in at least 30 minutes of exercise on at least five days a week. Various mechanisms have been proposed to explain how exercise may render sleep benefits. It is postulated that exercise increases energy expenditure and body temperature in a manner that facilitates sleep for recuperation of the body (McGinty \& Szymusiak, 1990; Adam \& Oswald, 1983; Berger \& Phillips, 1988). The anxiolytic and anti-depressant effects of exercise have also been described (Herring, O'Connor, \& Dishman, 2010; Singh, Clements, \& Fiatarone, 1997; Abedi, Nikkhah, \& Najar, 2015), in addition to improved immune functioning (Nehlsen-Cannarella, 1991) and QOL (Passos et al., 2011). Additionally, exercise may impact circadian rhythms, either through increasing light exposure (O'Connor \& Youngstedt, 1997), or serving as a Social Zeitgeber (German for time giver) (Ehlers, Frank, \& Kupfer, 1988). Finally, exercise may also exert its effects through providing distraction, improving self-efficacy, and providing the opportunity for social interaction and support networks (Peluso \& Andrade, 2005).

In combination with traditional physical exercise, the literature suggests that alternative mind-body interventions, incorporating stretching and flexibility exercises (e.g. yoga, tai chi), may also have beneficial effects for general health and sleep (Wang, Collet, \& Lau, 2004; Khalsa, 2004; Raman, Zhang, Minichiello, D’Ambrosio, \& Wang, 2013). It is important to note that these types of interventions have not been well evaluated as treatments for insomnia. It is postulated that mind-body exercise may have a different or additional mechanistic route to sleep improvement, beyond that of traditional physical exercise. Consistent with the hyperarousal model of insomnia 
(Bonnet, \& Arand, 1997), it is hypothesised that these multi-component practices may render sleep improvements by targeting arousal mechanisms that contribute to insomnia. Moreover, consistent with CBT, these alternative approaches involve focusing on the body and mind as an interconnected system (Raman et al., 2013). Such interventions emphasise control, mindfulness and meditation through the performance of repetitious, gentle, slow-paced movements and postures, combined with deep diaphragmatic breathing.

\section{Evidence base}

A significant body of research has investigated the effects of exercise on sleep. However, most studies have examined the cross-sectional association between selfreported exercise and sleep, which does not allow causation to be inferred; or, have explored the effects of exercise in experimental studies with healthy sleepers, which may underestimate the efficacy of exercise in promoting sleep. Although experimental investigations with individuals presenting with insomnia are scarce, exercise studies with poor sleepers do exist and have been examined in previous reviews.

A systematic review examining six exercise training trials found that long-term exercise (10-16 weeks) improved sleep quality, as measured using the PSQI, for middleaged and older adults with insomnia disorder and symptoms (Yang, Ho, Chen, \& Chien, 2012). Similarly, a 2017 review and meta-analysis of 5 RCTs examining programmed exercise (12-16 weeks) found that low-moderate levels of exercise significantly lowered the PSQI score for middle-aged women, whereas moderate aerobic exercise had a positive effect on sleep quality (Rubio-Arias, Marín-Cascales, Ramos-Campo, Hernandez, \& Pérez-López, 2017). Another systematic review with meta-analysis examined five studies targeting middle-aged and older adults (Passos et al., 2012). The results indicated that long-term exercise (16 weeks-12 months) led to improvements in objective and subjective measures of SOL and TST. Finally, a more recent meta-analysis of 66 studies, targeting individuals with and without sleep complaints (mean age 42.0 
years; range 18.3-88.5), reported that acute and regular exercise improved SQ and the following sleep continuity variables: TST, SOL, SE, and WASO (Kredlow, Capozzoli, Hearon, Calkins, \& Otto, 2015).

Whilst small to large treatment effects have been demonstrated, the validity and generalisability of these review findings is limited by several important factors. Chiefly, these reviews (i) lack standardised definition or measurement of sleep disturbance; (ii) examine a small number of trials, some predominated by small sample sizes; (iii) include limited trials with insomnia disorder; (iv) include studies where the sample also comprises healthy sleepers (e.g. Kredlow et al., 2015: only $11 \%$ of 66 reviewed studies recruited individuals presenting with sleep complaints); (v) include studies with inadequate control conditions (Passos et al., 2011) and; (vi) include studies with participants presenting with another primary disorder which was not a sleep disorder (i.e. a significant psychiatric comorbidity) (Singh et al., 1997). These factors have the potential to confound and affect the interpretation of results. The question of the sleeppromoting efficacy of exercise for insomnia is therefore yet to be clearly addressed through systematic review.

Since the publication of previous reviews, new evidence from exercise trials has become available. The methodological rigour of these studies has also increased, as evidenced by recruiting only poor sleepers and utilising randomised and controlled designs incorporating non-exercise control groups. To date, only one systematic review exists incorporating these new trials of exercise interventions for insomnia. Banno et al (2018) conducted a systematic review and meta-analysis on nine RCT's ( $n=557$ ) to examine the treatment effect of exercise in patients with primary and secondary insomnia. The predetermined exercise programs, consisting of any intensity, duration, and frequency had a beneficial effect on sleep according to the PSQI ( $8 w k-6 m: N=6$ (361); $M D=2.87 ; 95 \% \mathrm{Cl} 3.95$ to 1.79 ) and ISI (4-6mo: $\mathrm{N}=2(66) ; \mathrm{MD}=3.22 ; 95 \% \mathrm{Cl}$ 5.36 to 1.07$)$. However, exercise interventions were not associated with improved SOL, TST or SE. 
To the authors' knowledge, no review to date has i) focused exclusively on studies comprising whole samples of poor sleepers; ii) specified a minimum duration of physical activity as part of the study selection criteria; or iii) examined the treatment effects of exercise for those presenting with insomnia disorder and insomnia symptoms in isolation given their clinical heterogeneity.

$\underline{\text { Aims }}$

This review aimed to synthesize and critically appraise research evidence on the effects of exercise, at doses that approximate public health guidelines ( $>30$ minutes), for improving sleep in people with insomnia disorder and insomnia symptoms (not yet at the threshold of syndromal insomnia). The sleep-enhancing effects of exercise were systematically reviewed to address the following questions:

1. Does exercise improve sleep quality and subjective sleep parameters (i.e. as measured by validated questionnaire, sleep diary) for people with insomnia?

2. Does exercise improve objective sleep parameters (i.e. as measured by polysomnography, actigraphy) for people with insomnia?

\section{Method}

\section{Search Strategy}

This review was undertaken in compliance with PRISMA guidelines for the reporting of systematic reviews (The PRISMA Group, 2009). To identify eligible articles, one researcher $(\mathrm{HL})$ conducted searches using the electronic databases CINAHL plus, PsycINFO, EMBASE, MEDLINE, SPORTDiscus, and CENTRAL, accessed via the University 
of Manchester OVID and EBSCO online system between August 2016 and J anuary 2017. Two sets of search terms, relating to sleep and exercise, separated by "OR" were combined using the Boolean operator "AND". The search term combinations were: ("sleep disorder" OR "sleep disturbance" OR “chronic sleep problem" OR "sleep problem" OR “poor sleep" OR “chronic Insomnia" OR “insomnia*” OR "sleep complaint" OR "sleep quality") AND ("Exercise" OR "physical activity" OR "yoga” OR "tai chi” OR "qigong" OR “Pilates" OR “walking" OR "aerobic" OR “aquatic" OR "nondrug").

Inclusion criteria:

A. Randomised Controlled Trial (RCT).

B. Adults (>18 years).

C. Whole sample met criteria for insomnia (disorder or symptoms), as verified by diagnostic criteria, validated sleep measure, self-report, or objective measures in the sleep laboratory. For studies including samples with healthy sleepers, data had to be reported separately for poor sleepers.

D. Investigated >30-minute session of exercise per day, consisting of either moderate-vigorous intensity aerobic or resistance exercise, or mind-body exercise.

E. Investigated multiple sessions of exercise (chronic exercise training vs. acute exercise training).

F. Included a suitable, non-physically active control condition (i.e. non-/other intervention, wait-list control). 
G. Assessed sleep using subjective and/or objective validated measures of sleep outcome.

H. Peer reviewed paper published in English between 1950 and 2017.

Exclusion criteria:

A. Conference abstract, dissertation, letter, case study, or case series.

B. Investigated insomnia in the context of another medical or neurological condition (e.g. cancer; Parkinson's disease), psychiatric disorder (e.g. depression), or sleep disorder (e.g. sleep apnoea).

C. Examined atypical exercise regimens (e.g. extensive in duration ( $>4$ hour); during typical sleep hours).

D. Examined atypical sleep regimens (e.g. studies with shift workers; artificiallyinduced insomnia).

E. Contained a physically active comparison group only.

\section{Data Extraction}

Search results were exported to reference management software and duplicates were removed. Articles were screened based on title, abstract and full text by the first author $(\mathrm{HL})$, and a proportion were independently reviewed by another author $(\mathrm{AF}-\mathrm{H})$ to 
determine whether they met the eligibility criteria. Agreement between authors was good $($ Kappa $=0.848$ (title); 0.946 (abstract); 1.000 (full text), p <0.001). Inconsistencies were discussed and consensus was reached between researchers. Eligibility of the papers was finalised following review and discussion between all authors. Reference lists of eligible articles and reviews were then checked to identify additional articles. The selection process is shown in Figure 1.

Key demographic data were extracted from all eligible articles (see Tables 1-2). For studies that provided an age range rather than the mean, the median age was reported. The subjective and objective sleep outcomes were presented separately for insomnia disorder and insomnia symptoms (see Tables 3-6). 
Figure 1. PRISMA flow diagram of systematic search.

Records identified through
database searching
$(n=14,765)$

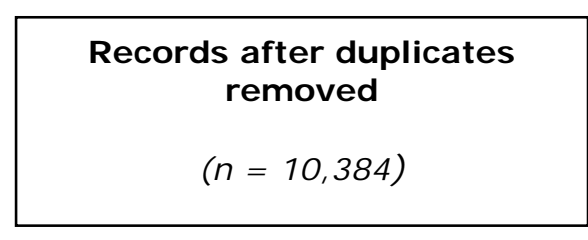

\begin{tabular}{c}
$\begin{array}{c}\text { Full-text articles retrieved for } \\
\text { eligibility } \\
(n=181)\end{array}$ \\
\hline
\end{tabular}

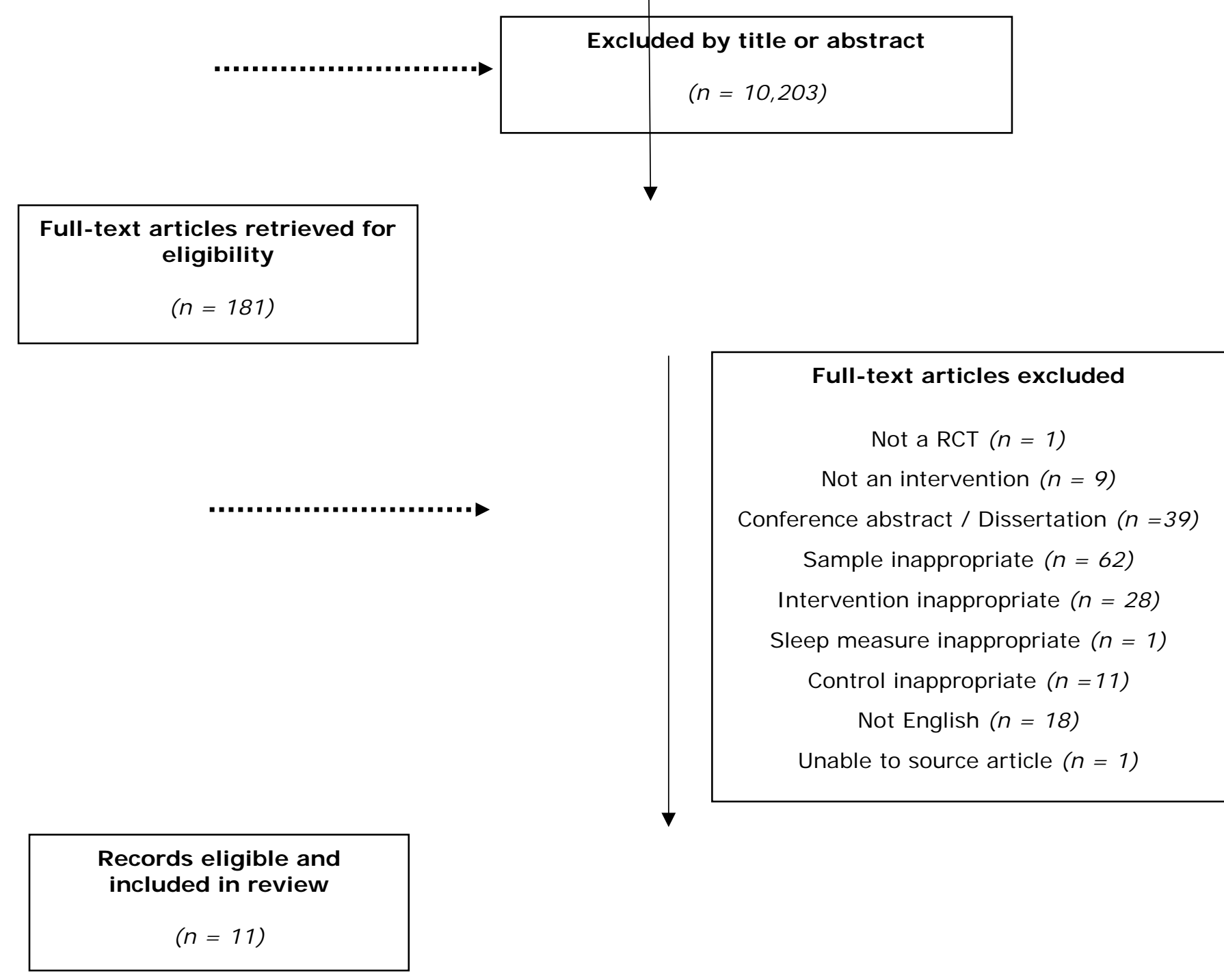




\section{Quality Assessment}

Existing quality assessment tools for clinical trials are criticised due to methodological inadequacies, which may potentially confound estimates of intervention efficacy (Jüni, Witschi, Bloch, \& Egger, 1999; Moher et al., 1995). The Clinical Trial Assessment Measure (CTAM) was chosen as it shows good reliability and concurrent validity (Tarrier \& Wykes, 2004). Individual features are drawn from CONSORT guidelines (Moher, Schulz, \& Altman, 2001), a review of 25 trial assessment scales [Moher et al., 1995), and expert opinions of mental health professionals and statistical experts, which together provide face validity.

The CTAM assesses studies on 15 items which are grouped into six methodological areas: 1) sample characteristics; 2) treatment allocation; 3) outcome assessment; 4) control groups; 5) treatment description, and; 6) analysis. The maximum score is 100 , with a score of $>65$ indicating adequate methodological quality (Wykes, Steel, Everitt, \& Tarrier, 2008). Item 11, regarding Treatment as Usual, was adapted for the purposes of the current review as this was not relevant to the current inclusion criteria. For a study to receive a maximum score on this item, the control group was required to be nonphysically active (Appendix A). Two authors (HL, AF) independently scrutinised the methodological quality of eligible studies using the CTAM. Any discrepancies were discussed and resolved by consensus.

\section{Assessment of heterogeneity}

Clinical, methodological, and statistical heterogeneity of eligible studies were assessed. If the studies were found to be homogenous, a meta-analysis would have been deemed appropriate. However, given the extensive heterogeneity of age, gender, sleep status, comparison groups, and measures used, and the inadequate methodological 
quality and reporting of many of the papers, a meta-analysis was considered unsuitable and potentially misleading. Narrative synthesis was therefore implemented to evaluate the quality and outcomes of the studies.

\section{Effect sizes}

Where sufficient data were available, the standardized mean difference (SMD) was calculated to express the size of the intervention effect relative to the specific sleep outcomes used within each study (Deeks, Higgins, \& Altman, editors; SMD = Difference in mean outcome between groups divided by the pooled standard deviation of outcome).

For studies employing validated questionnaires, effect sizes were calculated for the overall global score. For studies utilising sleep diaries and objective sleep measures, effect sizes were calculated for the main sleep continuity variables (SOL, WASO, TST, $\mathrm{SE})$. These were chosen as they are the most widely used measures of insomnia treatment effectiveness in clinical practice. For five studies, insufficient data were reported to permit calculation of the SMD. In these instances, the authors were contacted and additional data were obtained.

\section{Results}

\section{Study characteristics}

Eleven studies (Afonso et al., 2012; Guilleminault et al., 1995; Hartescu, Morgan, \& Stevinson, 2015; Irwin et al., 2014; Reid et al., 2010; Chan et al., 2016; Chen, Fox, Ku, \& Chang, 2016; Irwin et al., 2008; King, Oman, Brassington, Bliwise, \& Haskell, 1997; King et al., 2008; Tan, Alén, Wiklund, Partinen, \& Cheng, 2016) met inclusion criteria and were appraised in the review (see Tables 1-2). This included 10 RCTs and one pilot study with randomisation. 
Studies took place at various sites worldwide: six in the U.S, one in Brazil, two in Europe, one in Taiwan, and one in Hong Kong. Nine studies had between 30 and 70 participants, one had less than 25 participants, and one had over 120 participants. The total number of individuals participating and randomised in the included studies was 609 , whereas the total number of randomised participants included in the final analyses reduced to 581. Most studies included mixed-gender groups, one study included women only, and one study included men only. Overall, there was a greater proportion of women reported (408 female: 179 male*). The age of participants ranged from 30 to 97 years; the average age ranged from 44 to 80 years. Eight studies recruited older adults ( $>60$ years), whereas three studies targeted middle-aged adults (40-59 years).

\section{$\underline{\text { Sleep Status }}$}

Five studies (Afonso et al., 2012; Guilleminault et al., 1995; Hartescu et al., 2015; Irwin et al., 2014; Reid et al., 2010) included adults with insomnia disorder based on diagnostic criteria (American Psychiatric Association, 2013; American Academy of Sleep Medicine, 2005; Edinger et al., 2004).

Six studies included adults with insomnia symptoms. This was verified by: i) score of $>5$ on the PSQI (Buysse, Reynolds, Monk, Berman, \& Kupfer, 1989; Chan et al., 2016; Irwin et al., 2008); ii) subjective report in combination with <85\% sleep efficiency as determined by actigraphy (Frankel, Buchbinder, Coursey, \& Snyder, 1976; Coates et al., 1982; Chen at al., 2016); iii) ratings of $>3$ on $2 / 3$ items drawn from the Sleep Questionnaire and Assessment of Wakefulness (SQAW: Miles, 1982) which assesses problems of getting to sleep at night, waking up during the night, and waking and getting up in the morning, OR ratings of $>4$ on any $1 / 3$ of the same sleep items drawn from the SQAW (King et al., 1997; King et al., 2008), and; iv) meeting insomnia symptom criteria as verified by answers from the modified Basic Nordic sleep questionnaire (BNSQ) (Partinen \& Gislason, 1995; Tan et al., 2016). 


\section{Experimental conditions}

Seven studies examined aerobic exercise, and four studies examined mind-body exercise (Tai Chi, Yoga). Exercise duration was mostly between eight and 25 weeks, one study examined four weeks of exercise, and one study continued for 12 months. With the exclusion of one study (Reid et al., 2010), all participants engaged in at least 120 minutes of exercise per week. Only five studies (Guilleminault et al., 1995; Hartecsu et al., 2015; King et al., 1997; King et al., 2008; Tan et al., 2016) met the weekly guideline (150 minutes) specified by the WHO (2010).

Eight studies were two-arm trials with one intervention and one control arm. Three studies were three-arm trials which explored additional treatments (i.e. Cognitive Behavioural Therapy, light therapy, passive stretching). Five studies utilised a nonetreatment control group, whereas three used health education controls, two used sleep hygiene controls, and one combined sleep hygiene with recreational group activities (see Tables 1-2). Exercise was completed independently without supervision in three studies for insomnia disorder, whereas exercise was supervised on an individual or group basis for the remaining two studies. Exercise was supervised and group-based for all six studies for insomnia symptoms.

\section{Outcome measures}

Five studies reported both objective and subjective sleep measures, five reported subjective data only, and one study reported objective data only. In the objective domain, three studies used PSG (Afonso et al., 2012: 1 night at each of 2 timepoints (baseline, 4 months); Irwin et al., 2014: 3 nights at each of 2 timepoints (baseline, 4 months); King et al., 2008: 7 nights spread across 3 timepoints (baseline: 3 nights; 6 months: 2 nights; 12 months: 2 nights), two studies used actigraphy (Guilleminault et al., 1995: 2 nights at each of 2 timepoints (baseline, 5 weeks); Chen et al., 2016: 7 nights at each of 2 timepoints (baseline, posttreatment), and one used a sleep 
monitoring system which included a bed and ambient brightness sensor (Tan et al., 2016: 7 nights at each of 2 timepoints (baseline, posttreatment).

In the subjective domain, two studies used the Insomnia Severity Index (ISI: Bastien, Vallières, \& Morin, 2001), six used the PSQI, and one used the Athens insomnia Scale (AIS: Soldatos, Dikeos, \& Paparrigopoulos, 2000). Sleep diaries were used in five studies with one utilizing the Pittsburgh Sleep Diary (PSD: Monk et al., 1994).

Quality

An overview of quality rating for all studies is presented in Appendix B. Nine studies were categorised as being of adequate methodological quality, while two were rated below the recommended cut-off score $(>65)$. All studies included an appropriate control group who were encouraged to maintain baseline levels of physical activity, employed validated sleep measures, and adequately described the experimental conditions which was sufficient to permit replication. All but one of the studies monitored treatment adherence. Only two studies used objective measures (Pedometer, Portable Microprocessor) to measure treatment adherence, whereas most studies used subjective measures of exercise engagement (i.e. exercise logs, class attendance) and therefore, did not monitor activity levels beyond those recommended. The statistical methods in all studies were considered appropriate, although four did not use an intention to treat analysis (ITT) where this was required. Five studies were rated low for sample characteristics due to small and highly selective samples (i.e. volunteers) recruited primarily through advertisement, in addition to the absence of adequate a-priori power calculations. In most cases, quality ratings were reduced as the studies did not clearly report the randomisation process, or whether this was independent to the research team. Also, most studies did not specify whether assessments were carried out blind to group allocation where this was required (i.e. objective data scoring) or describe the method or verification process. 
Table 1. Summary of included studies for insomnia disorder

\begin{tabular}{|c|c|c|c|c|c|c|c|}
\hline \multirow[t]{2}{*}{ Reference } & \multirow[t]{2}{*}{ Location } & \multirow[t]{2}{*}{ Design } & \multirow{2}{*}{$\begin{array}{l}\text { Sample Group } \\
\text { characteristics } \\
\text { (sample size [included in } \\
\text { analysis], age, gender) }\end{array}$} & \multirow{2}{*}{$\begin{array}{l}\text { Duration and frequency } \\
\text { by Group }(\mathrm{G})\end{array}$} & \multirow{2}{*}{$\begin{array}{l}\text { Intervention } \\
\text { by Group (G) }\end{array}$} & \multicolumn{2}{|c|}{ Outcome measures } \\
\hline & & & & & & Objective & Subjective \\
\hline $\begin{array}{l}\text { Afonso et al., } \\
2012\end{array}$ & Brazil & $\mathrm{RCT}$ & $\begin{array}{l}\text { Postmenopausal women } \\
\text { with insomnia } \\
60[44], \mathrm{G} 1=15, \mathrm{G} 2= \\
14, \mathrm{G} 3=15 ; \text { median } \\
\text { age = } 57.5 \mathrm{y} ; \text { female only }\end{array}$ & $60 \mathrm{~min}$, twice/wk, $4 \mathrm{mo}$ & $\begin{array}{l}\text { G1: Yoga } \\
\text { G2: Passive stretching } \\
\text { G3: No treatment }\end{array}$ & $\frac{4 \mathrm{mo}}{\mathrm{PSG}}$ & $\frac{4 m o}{|S|}$ \\
\hline $\begin{array}{l}\text { Guilleminault } \\
\text { et al., } 1995\end{array}$ & US & $\mathrm{RCT}$ & $\begin{array}{l}\text { Psychophysiological } \\
\text { insomnia } \\
32[30], \mathrm{G} 1=10, \mathrm{G} 2= \\
10, \mathrm{G} 3=10 ; \text { mean age } \\
=44 \mathrm{y} ; 14 \text { males, } 18 \\
\text { females }\end{array}$ & $45 \mathrm{~min}, 7$ times/wk, $4 \mathrm{wk}$ & $\begin{array}{l}\text { G1: Structured sleep hygiene }+ \text { MAE } \\
\text { G2: Structured sleep hygiene + light } \\
\text { therapy } \\
\text { G3: Structured sleep hygiene }\end{array}$ & $\frac{\mathbf{5 w k}}{\text { Actigraphy }}$ & $\frac{\mathbf{5 w k}}{\text { Sleep log }}$ \\
\hline $\begin{array}{l}\text { Hartescu et al., } \\
2015\end{array}$ & UK & $\mathrm{RCT}$ & $\begin{array}{l}\text { PA people with } \\
\text { insomnia } \\
41[41], G 1=20, G 2= \\
21 ; \text { mean age }=60 \mathrm{y} ; \\
11 \text { males, } 30 \text { females }\end{array}$ & $30 \mathrm{~min}, 5$ times/wk, $6 \mathrm{mo}$ & $\begin{array}{l}\text { G1: MAE } \\
\text { G2: No treatment }\end{array}$ & & $\frac{6 \mathrm{mo}}{\mid \mathrm{SI}}$ \\
\hline $\begin{array}{l}\text { Irwin et al., } \\
2014\end{array}$ & US & $\mathrm{RCT}$ & $\begin{array}{l}\text { OA with chronic and } \\
\text { primary insomnia } \\
123[123], \mathrm{G} 1=48 \\
\mathrm{G} 2=50, \mathrm{G} 3=25 ; \mathrm{Age}= \\
55-85 \text { y; } 35 \text { males, } \\
88 \text { females }\end{array}$ & $120 \mathrm{~min}$, once/wk, $4 \mathrm{mo}$ & $\begin{array}{l}\text { G1: Tai chi } \\
\text { G2: CBT } \\
\text { G3: Sleep seminar }\end{array}$ & $\frac{4 \mathrm{mo}}{\mathrm{PSG}}$ & $\begin{array}{l}4,7 \& 16 \mathrm{mo} \\
\text { PSQI } \\
\text { AIS } \\
\text { PSD }\end{array}$ \\
\hline $\begin{array}{l}\text { Reid et al., } \\
2010\end{array}$ & US & $\mathrm{RCT}$ & $\begin{array}{l}\text { PA adults with } \\
\text { insomnia } \\
23[17], \mathrm{G} 1=10, \mathrm{G} 2= \\
7 ; \text { mean age }=61.6 \mathrm{y} \\
1 \text { male, } 16 \text { females }\end{array}$ & $\begin{array}{l}\text { G1: }(2 \times 20 \mathrm{~m} \text { OR } 1 \times 30- \\
40 \mathrm{~m}), 4 \text { times/wk, } 16 \mathrm{wk} \\
\text { G2: } 45 \mathrm{~m}, 3-5 \text { times } / w k \\
16 \mathrm{wk}\end{array}$ & 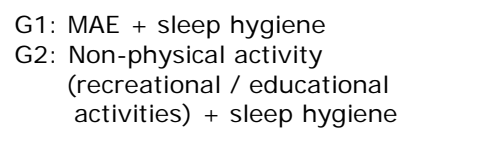 & & $\frac{16 w k}{P S Q I}$ \\
\hline
\end{tabular}

Abbreviations: AIS - Athens Insomnia Scale; CBT - Cognitive Behavioural Therapy; ISI - Insomnia Severity Index; MAE - Moderate-intensity aerobic exercise; OA - Older adult; PA - Physically inactive; PSD - Pittsburgh Sleep Diary; PSG - Polysomnography; PSQI - Pittsburgh Sleep Quality Index; RCT - Randomised controlled trial. 
Table 2. Summary of included studies for insomnia symptoms

\begin{tabular}{|c|c|c|c|c|c|c|c|}
\hline \multirow[t]{2}{*}{ Reference } & \multirow[t]{2}{*}{ Location } & \multirow[t]{2}{*}{ Design } & \multirow{2}{*}{$\begin{array}{l}\text { Sample Group } \\
\text { characteristics } \\
\text { (sample size [included in } \\
\text { analysis], age, gender) }\end{array}$} & \multirow{2}{*}{$\begin{array}{l}\text { Duration and frequency } \\
\text { by Group }(G)\end{array}$} & \multirow{2}{*}{$\begin{array}{l}\text { Intervention } \\
\text { by Group (G) }\end{array}$} & \multicolumn{2}{|l|}{ Outcome measures } \\
\hline & & & & & & Objective & Subjective \\
\hline $\begin{array}{l}\text { Chan et al., } \\
2016\end{array}$ & Hong Kong & Pilot RCT & $\begin{array}{l}\text { OA with sleep disturbance } \\
52[52], \mathrm{G} 1=27, \mathrm{G} 2= \\
25 ; \text { mean age }=80.6 \mathrm{y} \\
8 \text { males, } 44 \text { females }\end{array}$ & $\begin{array}{l}\text { G1: } 60 \mathrm{~m}, 2 \text { times/wk, } 2 \mathrm{mo} \\
\text { G2: once/wk, } 2 \mathrm{mo}\end{array}$ & $\begin{array}{l}\text { G1: Tai Chi } \\
\text { G2: Weekly health talk }\end{array}$ & & $\frac{2 \& 6 \mathrm{mo}}{\mathrm{PSQI}}$ \\
\hline $\begin{array}{l}\text { Chen et al., } \\
2016\end{array}$ & Taiwan & RCT & $\begin{array}{l}\text { OA with mild sleep } \\
\text { impairment } \\
67[63], G 1=29, G 2= \\
34 ; \text { mean age }=65.7 \mathrm{y} ; \\
13 \text { males, } 54 \text { females }\end{array}$ & $60 \mathrm{~min}, 2$ times/wk, 8wk & $\begin{array}{l}\text { G1: MAE } \\
\text { G2: No treatment }\end{array}$ & $\frac{9 w \mathbf{k}}{\text { Actigraphy }}$ & \\
\hline $\begin{array}{l}\text { Irwin et al., } \\
2008\end{array}$ & US & $\mathrm{RCT}$ & $\begin{array}{l}\text { OA with moderate sleep } \\
\text { complaints } \\
52[52], \mathrm{G} 1=30, \mathrm{G} 2= \\
22 ; \text { median age }=72.5 \text { y } \\
\text { y; } 16 \text { males, } 36 \text { females }\end{array}$ & $40 \mathrm{~m}, 3$ times/wk, $25 \mathrm{wk}$ & $\begin{array}{l}\text { G1: Tai chi } \\
\text { G2: Health education }\end{array}$ & & $\frac{16 \& 25 w k}{P S Q I}$ \\
\hline $\begin{array}{l}\text { King et al., } \\
1997\end{array}$ & US & RCT & $\begin{array}{l}\text { PA OA with } \\
\text { moderate sleep complaints } \\
48[48], \mathrm{G} 1=24, \mathrm{G} 2= \\
24 ; \text { median age }=63 \mathrm{y} ; \\
14 \text { males, } 34 \text { females }\end{array}$ & $\begin{array}{l}(60 \mathrm{~m} \text { class })+(40 \mathrm{~m} \\
\text { home-based), } 2 \text { times/ } \\
\text { wk, } 16 \mathrm{wk}\end{array}$ & $\begin{array}{l}\text { G1: MAE } \\
\text { G2: Wait-list control }\end{array}$ & & $\begin{array}{l}\frac{16 \mathbf{w k}}{\text { PSQI }} \\
\text { Sleep diary }\end{array}$ \\
\hline $\begin{array}{l}\text { King et al., } \\
2008\end{array}$ & US & RCT & $\begin{array}{l}\text { PA OA with mild- } \\
\text { moderate sleep complaints } \\
66[66], \mathrm{G} 1=36, \mathrm{G} 2= \\
30 ; \text { mean age }=61.4 \mathrm{y} ; \\
22 \text { males, } 44 \text { females }\end{array}$ & $\begin{array}{l}\text { G1: ( } 60 \mathrm{~m} \text { class, } 2 \text { times/ } \\
\text { wk) }+(30 \mathrm{~m} \text { home- } \\
\text { based exercise } 3 \text { times/ } \\
\text { wk), } 12 \mathrm{mo} \\
\text { G2: } 90 \mathrm{~m} \text {, once/ } \mathrm{wk}, 12 \mathrm{mo}\end{array}$ & $\begin{array}{l}\text { G1: MAE } \\
\text { G2: Health education }\end{array}$ & $\frac{12 \mathrm{mo}}{\mathrm{PSG}}$ & $\begin{array}{l}\frac{\mathbf{1 2 m o}}{\mathrm{PSQ}} \\
\text { Sleep diary }\end{array}$ \\
\hline $\begin{array}{l}\text { Tan et al., } \\
2016\end{array}$ & Finland & RCT & $\begin{array}{l}\text { Overweight and obese } \\
\text { men with chronic } \\
\text { Insomnia symptoms } \\
45[45], \mathrm{G} 1=24, \mathrm{G} 2= \\
21 ; \text { median age }=47.5 \mathrm{y} ; \\
\text { male only }\end{array}$ & $\begin{array}{l}30-60 \mathrm{~m}, 1-5 \text { times } / \mathrm{wk} \\
6 \mathrm{mo}\end{array}$ & $\begin{array}{l}\text { G1: Progressive aerobic } \\
\text { exercise according to } \\
\text { baseline fitness level } \\
\text { G2: No treatment }\end{array}$ & $\begin{array}{l}\frac{\mathbf{6 m o}}{\text { Sleep monitoring }} \\
\text { system including } \\
\text { a piezoelectric bed } \\
\text { bed } \& \text { ambient } \\
\text { brightness sensor }\end{array}$ & $\frac{6 \text { mo }}{\text { Sleep diary }}$ \\
\hline
\end{tabular}

Abbreviations: HAE - High-intensity aerobic exercise; MAE - Moderate-intensity aerobic exercise; OA - Older adult; PA - Physically inactive; PSG - Polysomnography; PSQI - Pittsburgh Sleep Quality Index; RCT - Randomised controlled trial. 
Table 3. Subjective sleep outcomes for insomnia disorder

\begin{tabular}{|c|c|c|c|c|c|c|c|}
\hline Reference & Group & Outcome & $\begin{array}{l}\text { Statistically } \\
\text { significant }\end{array}$ & SMD & Follow-up & $\begin{array}{l}\text { Statistically } \\
\text { significant }\end{array}$ & SMD \\
\hline $\begin{array}{l}\text { Afonso et al., } \\
2012\end{array}$ & $\begin{array}{l}\text { Yoga vs. } \\
\text { NT }\end{array}$ & ISI & $\checkmark$ & 0.86 & N/A & & \\
\hline \multirow[t]{3}{*}{$\begin{array}{l}\text { Guilleminault } \\
\text { et al., } 1995\end{array}$} & $\begin{array}{l}\text { SH + MAE vs. } \\
\text { SH }\end{array}$ & $\underline{\text { Sleep log }}$ & & & & & \\
\hline & & TST & $x$ & 0.07 & $\mathrm{~N} / \mathrm{A}$ & & \\
\hline & & $\mathrm{SOL}$ & $x$ & 0.31 & N/A & & \\
\hline $\begin{array}{l}\text { Hartescu et al., } \\
2015\end{array}$ & $\begin{array}{l}\text { MAE vs. } \\
\text { NT }\end{array}$ & ISI & $\checkmark$ & 0.58 & $\mathrm{~N} / \mathrm{A}$ & & \\
\hline \multirow{11}{*}{$\begin{array}{l}\text { I rwin et al., } \\
2014\end{array}$} & $\begin{array}{l}\text { TCC vs. } \\
\text { SC }\end{array}$ & PSQI & $\checkmark$ & 0.50 & $7 \mathrm{mo}$ & $\begin{array}{l}\checkmark \\
x\end{array}$ & 0.36 \\
\hline & & AIS & $\checkmark$ & 0.54 & $7 \mathrm{mo}$ & $\widehat{\checkmark}$ & 0.42 \\
\hline & & & & & $16 \mathrm{mo}$ & $x$ & 0.13 \\
\hline & & PSD & & & & & \\
\hline & & TST & $x$ & 0.02 & $\begin{array}{l}7 \mathrm{mo} \\
16 \mathrm{mo}\end{array}$ & $\begin{array}{l}x \\
x\end{array}$ & $\begin{array}{l}0.11 \\
0.08\end{array}$ \\
\hline & & SOL & $x$ & 0.03 & $7 \mathrm{mo}$ & $x$ & 0.05 \\
\hline & & & & & $16 \mathrm{mo}$ & $x$ & 0.16 \\
\hline & & SE & $x$ & 0.03 & $7 \mathrm{mo}$ & $x$ & 0.05 \\
\hline & & & & & $16 \mathrm{mo}$ & $x$ & 0.02 \\
\hline & & WASO & $x$ & 0.02 & $7 \mathrm{mo}$ & $x$ & 0.28 \\
\hline & & & & & $16 \mathrm{mo}$ & $x$ & 0.32 \\
\hline $\begin{array}{l}\text { Reid et al., } \\
2010\end{array}$ & $\begin{array}{l}\text { SH + MAE vs. } \\
\text { SH + REA }\end{array}$ & PSQI & $\checkmark$ & 2.55 & $\mathrm{~N} / \mathrm{A}$ & & \\
\hline
\end{tabular}

Abbreviations: AIS - Athens Insomnia Scale; ISI - Insomnia Severity Index; MAE - moderate-intensity aerobic exercise; N/A - Not applicable (No follow-up); NT - No treatment; PSD - Pittsburgh Sleep Diary; PSQI - Pittsburgh Sleep Quality Index; REA - Recreational and Educational Activities; SE - Sleep Efficiency; SH - Sleep Hygiene; SMD - Standardised Mean Difference (Effect Size); SOL - Sleep Onset Latency; SS - Sleep Seminar; TCC - Tai Chi; TST - Total Sleep Time; WASO Wake Time After Sleep Onset. 
Does exercise improve sleep quality and subjective sleep parameters for people with insomnia disorder?

Mind-body exercise:

Two studies evaluating the effect of mind-body exercises reported a significant improvement in sleep quality. The first study (Afonso et al., 2012) compared yoga with no treatment in middle-aged postmenopausal women and found that global ISI score significantly reduced in both groups at four months, but the reduction in the exercise group was significantly higher. This represented a large effect size (SMD $=0.86$ ) Improved QOL and resistance to stress was also observed for the exercise group.

Similarly, the second study (Irwin et al., 2014), comparing Tai Chi with a sleep seminar group in older adults, found that global PSQI score reduced in both groups at four months, but the reduction in the exercise group was significantly higher. This represented a medium effect size $(S M D=0.5)$. The AIS showed similar results $(S M D=$ $0.54)$. These improvements were maintained at seven months (PSQI $=0.36 ; \mathrm{AIS}=$ 0.42) which represented small to medium effect sizes, but the effects disappeared at 16 months. Coupled with this, improved depressive symptoms and risk of inflammation were observed for the exercise group. In contrast, the PSD did not detect a significant change in subjective parameters of sleep continuity.

Given that standardised questionnaires contain items on other constructs, which contribute to overall sleep quality (i.e. mood, functioning, distress), it is possible that these were being modified by exercise and not sleep directly. This may explain the absence of treatment effect for the PSD. The different populations and interventions could account for the differences in treatment effects between studies. Likewise, the control conditions may also account for these differences. Afonso et al., (2012) utilised a non-treatment control and reported a large effect size, whereas I rwin et al., (2014) utilised a sleep seminar education control group (over 16 sessions) and reported a medium effect size. However, the first study (Afonso et al., 2012) included a very small 
sample and lacked any reference to a power calculation or ITT despite many drop outs. These methodological concerns resulted in this study scoring below the cut-off on the CTAM, and therefore introduces uncertainty around the estimated effect size.

Aerobic exercise:

One study targeting middle-aged adults and examining moderate intensity aerobic exercise, did not detect group differences for subjective parameters of sleep continuity as measured using by sleep diary. This may genuinely reflect an absence of treatment effect which is plausible given the relatively short duration of the intervention (Guilleminault et al., 1995: four weeks). However, this could also reflect the methodological quality of the study as this did not score above the cut-off on the CTAM, mainly due to the small sample and absence of a power calculation.

In contrast, two studies evaluating moderate-intensity aerobic exercise of longer duration in physically inactive older adults detected significant improvements in sleep quality compared to the control group. The first study (Hartescu et al., 2015) found a significant reduction in global ISI score for the exercise group at six-month follow-up, but no significant change in the control group, which represented a medium effect size (SMD $=0.58)$. Reduced anxiety and depression scores were also observed for the exercise group. All significant effects were independent of light exposure.

The second study (Reid et al., 2010) found that global PSQI score for the exercise group significantly reduced at four months post-treatment [average 4.8 reduction, from $9.9( \pm 2.37)$ to $5.1( \pm 1.44)]$, with most of the group scoring 5 or below following treatment which is indicative of good overall sleep quality, whereas the control group showed increased scores (from $8.5( \pm 1.76)$ to $9.5( \pm 1.97)$. The SMD $(2.55)$, reflecting a very large effect size, appears to be very high. The exercise group also showed improved depressive symptoms and QOL, and sleep improvements remained after adjusting for depressive symptoms. 
It seems plausible to assume that the moderate to large effect sizes observed in both of these studies may be attributable to the study criteria, given that these studies recruited participants according to baseline exercise threshold (i.e. inactive people). Conversely the former two studies, which failed to detect treatment effects, did not reliably exclude individuals on basis of baseline physical activity levels. It is therefore possible that these individuals were already engaging in high levels of physical activity and may thus have less potential for change compared to those individuals deemed to be physically inactive.

Furthermore, these two studies utilised validated questionnaires which measure other constructs in addition to sleep quality (i.e. daytime impairment). Therefore, it may be these are modified by exercise and not sleep directly as measured by sleep diaries. Regardless of the mechanistic route of action underlying this relationship, it was demonstrated that exercise lessened the overall burden of insomnia and could thus serve as a potential target treatment.

Whilst the latter study (Reid et al., 2010) scored above the cut-off on the CTAM, the overall sampling characteristics did not achieve a quality score $(>0)$. Primarily, this study did not report a power calculation and also recruited a very small sample $(n=17)$. The large effect size may therefore be unreliable and should be interpreted with caution. As such, it seems rational to take heed of the findings of the former study (Hartescu et al., 2015) which received the highest overall rating for methodological quality. 
Table 4. Subjective sleep outcomes for insomnia symptoms

\begin{tabular}{|c|c|c|c|c|c|c|c|}
\hline Reference & Group & Outcome & $\begin{array}{l}\text { Statistically } \\
\text { significant }\end{array}$ & SMD & Follow-up & $\begin{array}{l}\text { Statistically } \\
\text { significant }\end{array}$ & SMD \\
\hline $\begin{array}{l}\text { Chan et al., } \\
2016\end{array}$ & $\begin{array}{l}\text { TCC vs. } \\
\text { HE }\end{array}$ & PSQI & $x$ & 0.15 & $6 \mathrm{mo}$ & $\checkmark$ & 0.62 \\
\hline $\begin{array}{l}\text { Irwin et al., } \\
2008\end{array}$ & $\begin{array}{l}\text { TCC vs. } \\
\text { HE }\end{array}$ & PSQI & $x$ & 0.33 & $25 w k$ & $\checkmark$ & 0.70 \\
\hline \multirow[t]{3}{*}{$\begin{array}{l}\text { King et al., } \\
1997\end{array}$} & $\begin{array}{l}\text { MAE vs. } \\
\text { NT }\end{array}$ & PSQI & $\checkmark$ & 1.26 & N/A & & \\
\hline & & Sleep diary & & & & & \\
\hline & & SOL & $\checkmark$ & 0.65 & N/A & & \\
\hline \multirow[t]{3}{*}{$\begin{array}{l}\text { King et al., } \\
2008\end{array}$} & $\begin{array}{l}\text { MAE vs. } \\
\text { HE }\end{array}$ & PSQI & $x$ & 0.07 & $\mathrm{~N} / \mathrm{A}$ & & \\
\hline & & Sleep diary & & & & & \\
\hline & & $\begin{array}{l}\text { SOL } \\
\text { TST }\end{array}$ & $\begin{array}{l}\checkmark \\
x\end{array}$ & $\begin{array}{l}0.10 \\
0.33\end{array}$ & $\begin{array}{l}\text { N/A } \\
\text { N/A }\end{array}$ & & \\
\hline \multirow[t]{2}{*}{$\begin{array}{l}\text { Tan et al., } \\
2016\end{array}$} & $\begin{array}{l}\text { PAE vs. } \\
\text { NT }\end{array}$ & $\underline{\text { Sleep diary }}$ & & & & & \\
\hline & & SOL & $x$ & 0.12 & N/A & & \\
\hline
\end{tabular}

Abbreviations: HE - Health Education; MAE - Moderate-intensity aerobic exercise; N/A - Not applicable (No follow-up); NT No treatment; PAE - Progressive aerobic exercise; PSQI - Pittsburgh Sleep Quality Index; SMD - Standardised Mean Difference (Effect Size); SOL - Sleep Onset Latency; TCC - Tai Chi; TST - Total Sleep Time. 
Does exercise improve sleep quality and subjective sleep parameters for people with insomnia symptoms?

Mind-body exercise:

Of the two studies evaluating the effect of Tai Chi in older adults, both found that exercise significantly improved sleep quality relative to the control group which received a health talk. The first study (I rwin et al., 2008) reported that global PSQI score significantly decreased for the exercise group when followed up at 25 weeks, whereas the control group instead showed an increase. The post-treatment effect size was medium to large $(S M D=0.70)$. A decline in depressive symptoms was also found in both conditions, indicating that these changes did not mediate sleep improvement. However, no significant improvement in global PSQI score was observed at the 16-week post-test interval.

Similarly, the second study (Chan et al., 2016) reported a significant improvement in global PSQI score for the exercise group at six-month follow-up, which represented a medium effect size $(S M D=0.62)$, but not two months immediately after completing the training class, although an improving trend was observed. The exercise group also reported improved QOL. Although both studies are of adequate methodological quality, the findings should still be interpreted with caution due to the small sample sizes.

Aerobic exercise:

Of the three studies evaluating the effects of aerobic exercise, two reported significant improvements for physically inactive older adults, whereas one did not detect treatment effects for middle-aged adults. The first study (Tan et al., 2016), exploring progressive aerobic exercise in middle-aged men, was long in duration (six months) but failed to detect a significant effect for SOL as measured using sleep diaries. This may 
genuinely reflect an absence of treatment effect, which is plausible given that the study was deemed to be of adequate methodological quality. However, these findings should still be interpreted with caution given the relatively small sample size, further compounded by the absence of any power calculation. The sampling characteristics for this study were subsequently rated as low in quality which introduces uncertainty around the effect size estimates observed.

One study which explored 12-month moderate-intensity aerobics in older adults did observe a significant improvement in SOL for the exercise group as measured using sleep diary (King et al., 2008). On the contrary, the control group instead showed an increase (and therefore deterioration) in SOL. This represented a small effect size (SMD $=0.1$. . However, sleep quality was not significantly improved as indicated by global PSQI score.

An earlier study by the same authors, which was shorter in duration (16 weeks) and received a higher rating for methodological quality (King et al., 1997), did find that exercise significantly improved global PSQI score. Whereas global PSQI score reduced for the intervention group, this remained relatively unchanged for the control group. This represented a very large effect size $(S M D=1.26)$. A significant treatment effect of medium size for SOL, as measured using sleep diary, was also observed at 16 weeks $(\mathrm{SMD}=0.65)$ but not eight weeks.

These differences may be attributable to the first study (King et al., 2008) including participants with mild to moderate sleep disturbance, whereas the latter (King et al., 1997) targeted those with moderate complaints which may be more amenable to change. Likewise, the control conditions may also account for these differences. The second study utilised a wait-list control and reported medium to large effect sizes (King et al., 1997), whereas the control group in King et al., (2008) received a health talk intervention - which may have some active ingredients - and reported a small effect size (King et al., 2008).

The small effect size reported for King et al., (2008) could also be attributed to baseline imbalances. Raw data demonstrate that there was a relatively large difference in 
SOL at baseline between the exercise and control group, whereby the exercise group presented with more severe SOL (group difference $=15.36$ minutes). Despite the significant reduction in SOL for the exercise group (38.44 vs. 26.02), the post-value for the exercise group still remained higher than the baseline value for the control group (23.08). Therefore, large within group change may indicate regression to the mean. 
Table 5. Objective sleep outcomes for insomnia disorder

\begin{tabular}{|c|c|c|c|c|}
\hline Reference & Group & Outcome & $\begin{array}{l}\text { Statistically } \\
\text { significant }\end{array}$ & SMD \\
\hline \multirow[t]{5}{*}{$\begin{array}{l}\text { Afonso et al., } \\
2012\end{array}$} & $\begin{array}{l}\text { Yoga vs. } \\
\text { NT }\end{array}$ & PSG & & \\
\hline & & TST & $x$ & 0.27 \\
\hline & & $\mathrm{SOL}$ & $x$ & 0.46 \\
\hline & & $\mathrm{SE}$ & $x$ & 0.47 \\
\hline & & WASO & $x$ & 0.20 \\
\hline \multirow[t]{3}{*}{$\begin{array}{l}\text { Guilleminault } \\
\text { et al., } 1995\end{array}$} & $\begin{array}{l}\mathrm{SH}+\mathrm{MAE} \text { vS. } \\
\mathrm{SH}\end{array}$ & Actigraphy & & \\
\hline & & TST & $x$ & 0.30 \\
\hline & & SOL & $x$ & 0.26 \\
\hline \multirow[t]{5}{*}{$\begin{array}{l}\text { Irwin et al., } \\
2014\end{array}$} & $\begin{array}{l}\text { TCC vs. } \\
\text { SS }\end{array}$ & PSG & & \\
\hline & & TST & $x$ & 0.05 \\
\hline & & SOL & $x$ & 0.15 \\
\hline & & SE & $x$ & 0.10 \\
\hline & & WASO & $x$ & 0.05 \\
\hline
\end{tabular}

Abbreviations: MAE - Moderate-intensity aerobic exercise; NT - No treatment; PSG - Polysomnography; SE - Sleep Efficiency; SH - Sleep hygiene; SMD - Standardised Mean Difference (Effect Size); SOL - Sleep Onset Latency; SS - Sleep seminar; TCC - Tai Chi; TST - Total Sleep Time; WASO - Wake Time After Sleep Onset. 
Does exercise improve objective sleep parameters for people with insomnia disorder?

Of the three studies using actigraphy (Guilleminault et al., 1995) and PSG (Afonso et al., 2012; Irwin et al., 2014) to evaluate the effect of mind-body and aerobic exercise on objective measures of sleep continuity, none detected significant group differences for older or middle-aged adults. This is despite two of the studies (Afonso et al., 2012; Irwin et al., 2014) finding significant effects for subjective sleep quality measured using validated sleep measures. It is appropriate to assume that these measures capture improvements in additional constructs beyond actual sleep, which contribute to the overall burden of insomnia and thus global measures of sleep quality.

The absence of reliable group differences may reflect the true absence of an effect of exercise on objective sleep parameters. This is plausible given the relatively short duration of one exercise intervention (Guilleminault et al., 1995: four weeks). Additionally, one study was of high methodological rigour and therefore had the potential to detect a true effect if it exists (Irwin et al., 2014). Moreover, two studies used PSG (the gold standard objective sleep measurement) yet failed to detect significant treatment effects (Afonso et al., 2012; Irwin et al., 2014).

However, it is important to note that two studies did not score above the cut-off for adequate methodological quality (Afonso et al., 2012; Guilleminault et al., 1995). Primarily, these studies were characterised by very small samples, lacked any reference to a power calculation, and did not reliably stipulate exclusion of participants based on baseline physical activity level. These studies may therefore have been underpowered to detect a true effect. 
Table 6. Objective sleep outcomes for insomnia symptoms

\begin{tabular}{|c|c|c|c|c|}
\hline Reference & Group & Outcome & $\begin{array}{l}\text { Statistically } \\
\text { significant }\end{array}$ & SMD \\
\hline \multirow[t]{5}{*}{$\begin{array}{l}\text { Chen et al., } \\
2016\end{array}$} & $\begin{array}{l}\text { MAE vs. } \\
\text { NT }\end{array}$ & Actigraph & & \\
\hline & & TST & $x$ & 0.03 \\
\hline & & SOL & $\checkmark$ & 0.95 \\
\hline & & SE & $\checkmark$ & 0.79 \\
\hline & & WASO & $x$ & 0.41 \\
\hline \multirow[t]{4}{*}{$\begin{array}{l}\text { King et al. } \\
2008\end{array}$} & $\begin{array}{l}\text { MAE vs. } \\
\text { HE }\end{array}$ & & & \\
\hline & & TST & $x$ & 0.21 \\
\hline & & SOL & $x$ & 0.33 \\
\hline & & SE & $x$ & 0.11 \\
\hline \multirow[t]{5}{*}{$\begin{array}{l}\text { Tan et al., } \\
2016\end{array}$} & $\begin{array}{l}\text { PAE vs. } \\
\text { NT }\end{array}$ & \multicolumn{3}{|c|}{$\begin{array}{l}\text { Sleep monitoring system } \\
\text { (including a piezoelectric } \\
\text { bed } \& \text { ambient brightness } \\
\text { sensor) }\end{array}$} \\
\hline & & TST & $x$ & 0.39 \\
\hline & & SOL & $\checkmark$ & 0.31 \\
\hline & & SE & $x$ & 0.28 \\
\hline & & WASO & $x$ & 0.07 \\
\hline
\end{tabular}

Abbreviations: HE - Health education; MAE - Moderate-intensity aerobic exercise; NT - No treatment; SE - Sleep Efficiency; SMD - Standardised Mean Difference (Effect Size); SOL - Sleep Onset Latency; PAE - Progressive aerobic exercise; PSG Polysomnography; TST - Total Sleep Time; WASO - Wake Time After Sleep Onset. 
Does exercise improve objective sleep parameters for people with insomnia symptoms?

Of the three studies using different methodologies (PSG, actigraphy, Sleep monitoring system) to examine aerobic exercise, only two detected a significant improvement in objective parameters of sleep continuity. The first study, using PSG to explore moderate-intensity aerobic exercise for physically inactive older adults (King et al., 2008), did not detect any significant differences for sleep continuity variables. This is despite finding a significant effect for subjective SOL.

On the contrary, one study (Tan et al., 2016) which used a sleep monitoring system to explore change throughout progressive aerobic exercise in middle-aged men, found that SOL decreased for exercisers at six-month follow-up, whereas the control group showed an increase. This represented a small effect size (SMD $=0.31$ ). Simultaneously, WASO and SE showed a trend toward improvement although this did not meet statistical significance.

Similarly, the second study using actigraphy to explore moderate-intensity aerobic exercise in older adults (Chen et al., 2016) also found that SOL decreased for the exercise group at eight-week follow-up, whereas SOL increased for the control group. This represented a large effect size $(S M D=0.95)$. Moreover, a significant treatment effect was observed for SE; both groups showed an increase but this was more pronounced for the exercise group (SMD $=0.79$ ) which represented a large effect size. No significant differences were observed for the other sleep continuity variables (TST, WASO), although an improving trend was observed.

Unlike the latter two studies (Chen at al., 2016; Tan et al., 2016) which utilised no treatment control groups and reported small to large effect sizes, King et al., 2008 enrolled control participants into a health talk intervention and failed to detect treatment effects. This suggests that the control conditions may potentially account for differences in treatment effects.

Whilst all studies were considered to be of adequate methodological quality, these conflicting findings should be interpreted with caution. This is due to concerns regarding 
small samples, absence of power calculations, and many statistical tests being conducted. Also, for the two studies reporting significant results, one (Tan et al., 2016) relied upon sleep measurements carried out by technology with limited or no validation, and the other (Chen et al., 2016) exhibited large group differences at baseline with respect to SOL. The absence of rater blinding in the latter study (Chen et al., 2016) may also explain the large effect sizes observed. Together, these factors introduce uncertainty around the effect size estimates.

\section{Discussion}

\section{Main Findings}

This paper systematically reviewed the relationship between exercise and sleep in the context of insomnia. Statistically significant improvements in sleep quality, as indicated by validated questionnaires, were observed in all four studies targeting individuals with insomnia disorder. Similarly, three out of four studies targeting individuals with insomnia symptoms demonstrated significant improvements in sleep quality. Sleep diaries failed to detect significant treatment effects for sleep continuity variables in two studies targeting individuals with insomnia disorder. On the other hand, SOL was significantly improved in two out of three studies targeting individuals with insomnia symptoms.

Despite significant effects being observed for subjective sleep outcomes, exercise was not shown to improve objective parameters of sleep continuity in any of the three studies targeting individuals with insomnia disorder. In comparison, aerobic exercise was found to significantly improve objective parameters of sleep continuity in two out of three studies recruiting individuals with insomnia symptoms. Both studies detected improvements in SOL, whereas only one reported significant improvements in SE. While these inferences are drawn from findings of very few studies, some with low quality 
ratings, objective findings are consistent with sleep diary data: treatment effects were observable only for those presenting with insomnia symptoms. Validated questionnaires measure additional constructs which contribute to overall sleep quality (e.g. daytime impairment). Therefore, exercise could potentially exert its effects through these constructs rather than impacting sleep directly. This may explain the conflicting results between the validated questionnaires and both objective and subjective measures of sleep continuity for individuals presenting with insomnia disorder. Moreover, stronger exercise effects on sleep parameters in those with insomnia symptoms vs disorder may also suggest that chronic poor sleep - perhaps with a stronger psychological or behavioural basis - may be more resistant to change with exercise.

Of note the nature of the control condition appeared to influence outcomes, with larger group differences observed in studies which utilised a wait-list control group, receiving no form of intervention or contact. It could be hypothesised that for those studies whereby the control group did receive some form of intervention (e.g. sleep seminar education, sleep hygiene, health talk, group recreational activities), participants derived some benefit through non-specific factors (e.g. expectation, social contact, group support, directed attention etc). Alternatively, it may be that waitlist controls operate similar to nocebo conditions (Furukawa et al., 2014).

Whilst positive findings have been observed in the current review, it is considerably difficult to make any firm conclusions or generalisations regarding the efficacy of exercise for those presenting with insomnia. The literature to date is dominated by studies which are marred by methodological limitations. More than one third of studies were considered low in methodological quality, primarily due to recruiting small and highly selective samples, and the absence of power calculations. Being underpowered reduces the chance of detecting a true effect and reduces the likelihood that a statistically significant result reflects a true effect. This is a common issue encountered in psychology and neuroscience, and something which requires careful consideration in future studies (Button et al., 2013). There was also a lack of consistency and standardization in the definition and assessment of insomnia, and in the outcome 
measures used across studies, which may account for the lack of consistency in outcomes. Very few studies examined both objective and subjective sleep measurement, and few implemented PSG or sleep diaries which are recognised as the gold standard sleep measures. The treatment effects could also be explained by uncontrolled variables as most studies did not adequately screen for the presence of other sleep disorders, or control for other factors which may potentially impact sleep (i.e. substance use, caffeine). Additionally, only four out of eleven included studies stipulated study inclusion on the grounds of baseline physical activity levels, which is likely to contribute to the variability in observed treatment effects.

\section{Strength and limitations}

To the author's knowledge this is the first systematic review to investigate the sleep-enhancing effect of exercise by focusing exclusively on 1) controlled trials comprising poor sleepers only, and; 2) exercise interventions that are consistent with national guidelines. The review also employed a rigorous systematic approach, using highly selective criteria for the identification, screening and appraisal of relevant literature. Additionally, the SMD was calculated as a measure of effect size. This enabled comparisons to be made between studies exploring different populations, interventions and outcomes.

Several limitations should be considered. Generalisability of conclusions is limited by the relatively small sample sizes, study location, and preponderance of women and older people. The review also focused predominantly on mind-body and moderateintensity aerobic exercise. Comparisons between ages and genders, and different modes and intensity of exercise was beyond the scope of this review. Additionally, given the inadequate reporting in most studies regarding exercise characteristics (i.e. location, timing, supervision, individual vs. group-based), it was not possible to establish whether these variables influence outcome. The current review also did not specify the type of insomnia (sleep initiation or maintenance) to be investigated or duration of the condition, 
therefore comparisons between different patient groups were not possible. We also excluded studies investigating sleep in the context of another primary condition which was not insomnia, as these conditions can have a large impact on sleep (Ohayon, \& Roth, 2003; Roth, 2007). This was deemed important because we examined the sleepenhancing effect of exercise without the confounding influence of these conditions however we realise that insomnia often presents with comorbidities.

For the purpose of this review, we only focused on outcomes derived from validated sleep measures and objective and subjective measures of sleep continuity, to the exclusion of architectural sleep parameters. Therefore, a systematic review of the relationship between exercise and sleep architectural variables may be undertaken as the field continues to develop. Whilst the review investigated exercise for insomnia disorder and insomnia symptoms separately, it is possible that those with symptoms may meet criteria for disorder but are yet to be properly assessed. As such, findings should be interpreted with this in mind. Whilst this review endeavored to explore exercise interventions consistent with national guidelines (i.e. dose of 30 minutes / day), the authors did not use the weekly recommendation (150 minutes) as part of their eligibility criteria. Of note, this would have delivered only six studies. Finally, whilst the SMD is a recommended measure of effect size, baseline imbalances present for some studies may engender gross discrepancies between statistical significance and effect size magnitude.

\section{Implications for research and practice}

Given the limitations of the existing research, this review highlights several important areas in need of further research to help understand the relationship between exercise and sleep in insomnia. First, there is a necessity for well-controlled and methodologically robust studies to be conducted in people presenting with insomnia disorder and symptoms, across the age-range. These future studies should apply strict criteria to the assessment and diagnosis of insomnia and employ high-resolution measurements of sleep parameters and intervention adherence. Future studies should 
pay close to attention to sample size and statistical power, in addition to baseline physical activity levels, when designing clinical trials and assess the extent to which change in activity mediates improvement in sleep. Duration and type of insomnia may also affect the response to exercise training. Studies may wish to directly compare exercise for groups with different sleep profiles (i.e. chronic insomnia disorder vs. insomnia symptoms vs. healthy sleepers) and consider the impact of exercise characteristics (i.e. mode, intensity, dose, duration, supervision, timing, location) on sleep outcomes.

Finally, the field requires studies designed to uncover mechanisms of action responsible for sleep enhancement. This point is particularly relevant to mind-body therapies. These multi-component approaches, incorporating mind and body elements, could work through both exercise and mindfulness, or mindfulness could possibly enhance the effects of exercise. For example, some studies exploring Tai Chi have demonstrated that improvements in sleep are independent of the physical activity component (Li, Fisher, Harmer, Irbe, Tearse, \& Weimer, 2004; Irwin, Olmstead, $\&$ Motivala, 2008). Mechanistic trials with embedded measurement of putative mediators are clearly necessary.

\section{Conclusion}

Based on review findings we tentatively conclude that exercise can have a beneficial effect on sleep, with differential treatment effects being observed for insomnia disorder and insomnia symptoms. The clinical implication is that physical exercise could represent an alternative or complementary approach to existing therapies for sleep problems. Moreover, physical exercise could potentially be used as a preventative stepped-care approach in clinical practice, to target prodromal insomnia symptoms before the onset of clinically significant, chronic insomnia disorder. Given the small 
literature and methodological limitations present, future high-quality and well-controlled studies are needed before firm conclusions can be made. 


\section{References}

Abedi, P., Nikkhah, P., \& Najar, S. (2015). Effect of pedometer-based walking on depression, anxiety and insomnia among postmenopausal women. Climacteric, 18(6), 841-845.

Adam, K., \& Oswald, I. (1983). Protein synthesis, bodily renewal and the sleep-wake cycle. Clinical Science, 65(6), 561-567.

Afonso, R. F., Hachul, H., Kozasa, E. H., Oliveira, D. de S., Goto, V., Rodrigues, D., Tufik, S., \& Leite, J. R. (2012). Yoga decreases insomnia in postmenopausal women: a randomized clinical trial. Menopause, 19(2), 186-193.

Aikens, J. E., \& Rouse, M. E. (2005). Help-seeking for insomnia among adult patients in primary care. The J ournal of the American Board of Family Medicine, 18(4), 257-261.

American Academy of Sleep Medicine. (2005). ICSD-2 - International classification of sleep disorders: Diagnostic and coding manual. ( $2^{\text {nd }}$ ed.). Westchester IL: American Academy of Sleep Medicine.

American Psychiatric Association. (2013). Diagnostic and Statistical Manual of Mental Disorders (5th ed.). Arlington, VA: American Psychiatric Publishing.

Banno, M., Harada, Y., Taniguchi, M., Tobita, R., Tsujimoto, H., Tsujimoto, Y., ... Noda, A. (2018). Exercise can improve sleep quality: a systematic review and metaanalysis. PeerJ 6: e5172; DOI 10.7717/peerj.5172

Bastien, C. H., Vallières, A., \& Morin, C. M. (2001). Validation of the Insomnia Severity Index as an outcome measure for insomnia research. Sleep Medicine, 2(4), 297-307.

Benca, R. M. (2005). Diagnosis and treatment of chronic insomnia: A review. Psychiatric Services, 56(3), 332-343.

Berger, R. J., \& Phillips, N. H. (1988). Comparative aspects of energy metabolism, body temperature and sleep. Acta Physiologica Scandinavica, 133(574), 21-28.

Bolge, S. C., Doan, J. F., Kannan, H., \& Baran, R. W. (2009). Association of insomnia with quality of life, work productivity, and activity impairment. Quality of Life Research, 18(4), 415-422. Bonnet, M.H., \& Arand, D.L. (1997). Hyperarousal and insomnia. Sleep Medicine Reviews, 1(2), 97-108. 
Buman, M. P., \& King, A. C. (2010). Exercise as a treatment to enhance sleep. American J ournal of Lifestyle Medicine, 4(6), 500-514.

Button, K. S., Ioannidis, J. P., Mokrysz, C., Nosek, B. A., Flint, J., Robinson, E. S., \& Munafò, M. R. (2013). Power failure: why small sample size undermines the reliability of neuroscience. Nature Reviews Neuroscience, 14(5), 365-376.

Buysse, D.J., Reynolds, C. F., Monk, T. H., Berman, S. R., \& Kupfer, D. J. (1989). The Pittsburgh sleep quality index: a new instrument for psychiatric practice and research. Psychiatry Research, 28(2), 193-213.

Chan, A. W., Yu, D. S., Choi, K. C., Lee, D. T., Sit, J. W., \& Chan, H. Y. (2016). Tai chi qigong as a means to improve night-time sleep quality among older adults with cognitive impairment: a pilot randomized controlled trial. Clinical Interventions in Aging, 11, 1277-1286.

Chen, L. J., Fox, K. R., Ku, P. W., \& Chang, Y. W. (2016). Effects of Aquatic Exercise on Sleep in Older Adults with Mild Sleep Impairment: A Randomized Controlled Trial. International J ournal of Behavioral Medicine, 23(4), 501-506.

Coates, T. J., Killen, J. D., George, J., Marchini, E. W., Silverman, S., \& Thoresen, C. E. (1982). Estimating sleep parameters: a multitrait-multimethod analysis. Journal of Consulting and Clinical Psychology, 50(3), 345-352.

Colten, H. R., \& Altevogt, B. M. (2006). Sleep Disorders and Sleep Deprivation: An Unmet Public Health Problem. Washington, DC: National Academies Press.

Daley, M., Morin, C. M., LeBlanc, M., Gregoire, J. P. \& Savard, J. (2009). The economic burden of insomnia: direct and indirect costs for individuals with insomnia syndrome, insomnia symptoms, and good sleepers. Sleep, 32(1), 55-64.

Deeks, J. J., Higgins, J. P. T., \& Altman, D. G. (editors). Chapter 9: Analysing data and undertaking meta-analyses. In: Higgins, J. P. T., \& Green, S. (editors). Cochrane Handbook for Systematic Reviews of Interventions Version 5.1.0 (updated March 2011). The Cochrane Collaboration, 2011. Available from www.handbook.cochrane.org.

Driver, H. S., \& Taylor, S. R. (2000). Exercise and sleep. Sleep Medicine Reviews, 4(4), 387-402. Edinger, J. D., Bonnet, M. H., Bootzin, R. R., Doghramji, K., Dorsey, C. M., Espie, C. A., Jamieson, A. O., McCall, W. V., Morin, C. M., \& Stepanski, E. J. (2004). Derivation of 
research diagnostic criteria for insomnia: report of an American Academy of Sleep Medicine Work Group. Sleep, 27(8), 1567-1596.

Ehlers, C. L., Frank, E., \& Kupfer, D. J. (1988). Social zeitgebers and biological rhythms. Archives of General Psychiatry, 45(10), 948-952.

Espie, C. (2006). Overcoming Insomnia and Sleep Problems: A Self-Help Guide Using Cognitive Behavioral Techniques. London: Robinson.

Frankel, B. L., Buchbinder, R., Coursey, R. D., \& Snyder, F. (1976). Recorded and reported sleep in primary chronic insomnia. Archives of General Psychiatry, 33(5), 615-623. Furukawa, T. A., Noma, H., Caldwell, D. M., Honyashiki, M., Shinohara, K., Imai, H., Chen, P., Hunot, V., \& Churchill, R. (2014). Waiting list may be a nocebo condition in psychotherapy trials: a contribution from network meta-analysis. Acta Psychiatrica Scandinavica, 130(3), 181- 92.

Guilleminault, C., Clerk, A., Black, J, Labanowski, M., Pelayo, R., \& Claman, D. (1995). Nondrug treatment trials in psychophysiologic insomnia. Archives of Internal Medicine, 155(8), 838844.

Hartescu, I., Morgan, K., \& Stevinson, C. D. (2015). Increased physical activity improves sleep and mood outcomes in inactive people with insomnia: a randomized controlled trial. Journal of Sleep Research, 24(5), 526-534.

Harvey, A. G. (2001). Insomnia: symptom or diagnosis? Clinical Psychology Review, 21(7), $1037-\quad 1059$.

Herring, M. P., O'Connor, P. J., \& Dishman, R. K. (2010). The Effect of Exercise Training on Anxiety Symptoms Among Patients: A Systematic Review. Archives of Internal Medicine, $170(4), 321-\quad 331$.

Hohagen, F., Rink, K., Kappler C., Schramm, E., Riemann, D., Weyerer, S., \& Berger, M. (1993).

Prevalence and treatment of insomnia in general practice. A longitudinal study. European Archives of Psychiatry and Clinical Neurosciences, 242(6), 329-336.

Irish, L. A., Kline, C. E., Gunn, H. E., Buysse, D. J., \& Hall, M. H. (2015). The Role of Sleep Hygiene in Promoting Public Health: A Review of Empirical Evidence. Sleep Medicine Reviews, 22, 23- 36. 
Irwin, M. R., Olmstead, R., Carrillo, C., Sadeghi, N., Breen, E. C., Witarama, T., Yokomizo, M., Lavretsky, H., Carroll, J. E., Motivala, S.J., Bootzin, R., \& Nicassio, P. (2014). Cognitive behavioral therapy vs. Tai Chi for late life insomnia and inflammatory risk: a randomized controlled comparative efficacy trial. Sleep, 37(9), 1543-1552.

Irwin, M. R., Olmstead, R., \& Motivala, S. J. (2008). Improving sleep quality in older adults with moderate sleep complaints: A randomized controlled trial of Tai Chi Chih. Sleep, 31(7), 10011008.

Jüni, P., Witschi, A., Bloch, R., \& Egger, M. (1999). The hazards of scoring the quality of clinical trials for meta-analysis. The J ournal of the American Medical Association, 282(11), 1054-60.

Kaplan, H., Thompson, R. C., Trumble, B. C., Wann, L. S., Allam, A. H., Beheim, B., Frohlich, B., Sutherland, M. L., Sutherland, J. D., Stieglitz, J., Rodriguez, D. E., Michalik, D. E., Rowan, C. J., Lombardi, G. P., Bedi, R., Garcia, A. R., Min, J. K., Narula, J., Finch, C. E., Gurven, M., \& Thomas, G. S. (2017, April 29). Coronary Atherosclerosis in Indigenous South American Tsimane: A Cross-Sectional Cohort Study. The Lancet, 389(10080), 1730-1739.

Khalsa, S. B. (2004). Treatment of chronic insomnia with yoga: a preliminary study with sleepwake diaries. Applied Psychophysiology and Biofeedback, 29(4), 269-78.

King, A. C., Oman, R. F., Brassington, G. S., Bliwise, D.L, \& Haskell, W. L. (1997). Moderate-intensity exercise and self-rated quality of sleep in older adults. A randomized controlled trial. J ournal of the American Medical Association, 277(1), 32-7.

King, A. C., Pruitt, L A., Woo, S., Castro, C. M., Ahn, D. K., Vitiello, M. V., Woodward, S. H., \& Bliwise, D. L. (2008). Effects of moderate-intensity exercise on polysomnographic and subjective sleep quality in older adults with mild to moderate sleep complaints. The Journals of Gerontology, Series A: Biological Sciences and Medical Sciences, 63(9), 997-1004.

Kredlow, M. A., Capozzoli, M. C., Hearon, B. A., Calkins, A. W., \& Otto, M. W. (2015). The effects of physical activity on sleep: a meta-analytic review. Journal of Behavioral Medicine, 38(3), 427-449. 
Kripke, D. F., Garfinkel, L., Wingard, D. L., Klauber, M. R., \& Marler, M. R. (2002) Mortality associated with sleep duration and insomnia. Archives of General Psychiatry, 59(2), 131-136.

Kyle, S. D., Morgan, K., \& Espie, C. A. (2010). Insomnia and health-related quality of life. Sleep Medicine Reviews, 14(1), 69-82.

Li, F., Fisher, K.J., Harmer, P., Irbe, D., Tearse, R.G., \& Weimer, C. (2004). Tai chi and self-rated quality of sleep and daytime sleepiness in older adults: a randomized controlled trial. Journal of the American Geriatrics Society, 52(6), 892-900.

McGinty, D., \& Szymusiak, R. (1990). Keeping cool, a hypothesis about the mechanisms and functions of slow-wave sleep. Trends in Neurosciences, 13(12), 480-487.

Miles, L. E. (1982). A sleep questionnaire. In C. Guilleminault (Ed.), Sleeping and Waking Disorders: Indications and Techniques (pp. 383-413). Menlo Park, CA: Addison-Wesley Publishing Company, Medical/Nursing Division.

Moher, D., Jadad, A. R., Nichol, G., Penman, M., Tugwell, P., \& Walsh, S. (1995). Assessing the quality of randomized controlled trials: an annotated bibliography of scales and checklists. Controlled Clinical Trials, 16(1), 62-73.

Moher, D., Schulz, K. F., \& Altman, D. G. (2001). The CONSORT statement: revised recommendations for improving the quality of reports of parallel-group randomised trials. The Lancet, 357(9263), 1191-4.

Monk, T. H., Reynolds, C. F., Kupfer, D. J., Buysse, D. J., Coble, P. A., Hayes, A. J., Machen, M. A., Petrie, S. R., \& Ritenour, A. M. (1994). The Pittsburgh Sleep Diary. Journal of Sleep Research, 3(2), 111-20.

Morin, C. M., Belleville, G., Bélanger, L., \& Ivers, H. (2011). The Insomnia Severity Index: Psychometric Indicators to Detect Insomnia Cases and Evaluate Treatment Response. Sleep, 34(5), 601-608.

Morin, C. M., \& Benca, R. (2012). Chronic insomnia. The Lancet, 379(9821), 1129-1141.

Morin, C. M., Culbert, J. P., \& Schwartz, S. M. (1994). Nonpharmacological interventions for insomnia: a meta-analysis of treatment efficacy. The American J ournal of Psychiatry, 151(8), 1172-1180. 
National Center for Complementary and Alternative Medicine, (2011). Exploring the Science of Complementary and Alternative Medicine: NCCAM Third Strategic Plan 2011-2015. Retrieved February 13, 2018 from https://nccih.nih.gov/sites/nccam.nih.gov/files/NCCAM_SP_508.pdf National Sleep Foundation (n.d.). Healthy Sleep Tips. Retrieved October 10, 2016 from https://sleepfoundation.org/sleep-tools-tips/healthy-sleep-tips

Nehlsen-Cannarella, S. L., Nieman, D. C., Balk-Lamberton, A. J., Markoff, P. A., Chritton, D. B., Gusewitch, G., \& Lee, J. W. (1991). The effects of moderate exercise training on immune response. Medicine \& Science in Sports \& Exercise, 23(1), 64-70.

O'Connor, P. J., \& Youngstedt, S. D. (1997). Sleep quality in older adults: effects of exercise training and influence of sunlight exposure. Journal of the American Medical Association, 277(13), 1034-1035.

Ohayon, M. M. (2002). Epidemiology of insomnia: what we know and what we still need to learn. Sleep Medicine Reviews, 6(2), 97-111.

Ohayon, M. M., \& Roth, T. (2003). Place of chronic insomnia in the course of depressive and anxiety disorders. Journal of Psychiatric Research, 37(1), 9-15.

Paffenbarger, R. S., J r., Hyde, R. T., Wing, A. L., Lee, I. M., Jung, D. L., \& Kambert, J. B. (1993). The association of changes in physical-activity levels and other lifestyle characteristics with mortality among men. The New England J ournal of Medicine, 328(8), 538-545.

Partinen, M., \& Gislason, T. (1995). Basic Nordic Sleep Questionnaire (BNSQ): a quantitated measure of subjective sleep complaints. Journal of Sleep Research, 4(s1), 150-155.

Passos, G. S., Poyares, D., Santana, M. G., D’Aurea, C. V. R., Youngstedt, S. D., Tufik, S., \& de Mello, M. T. (2011). Effects of moderate aerobic exercise training on chronic primary insomnia. Sleep Medicine, 12(10), 1018-1027.

Passos, G. S., Poyares, D. L. R., Santana, M. G., Tufik, S., \& de Mello, M. T. (2012). Is exercise an alternative treatment for chronic insomnia? Clinics, 67(6), 653-659.

Peluso, M. A., \& Andrade, L. H. (2005). Physical activity and mental health: the association between exercise and mood. Clinics, 60(1), 61-67. 
Penedo, F. J., \& Dahn, J. R. (2005). Exercise and well-being: a review of mental and physical health benefits associated with physical activity. Current Opinion in Psychiatry, 18(2), 189193.

Raman, G., Zhang, Y., Minichiello, V. J., D’Ambrosio, C., \& Wang, C. (2013). Tai Chi Improves Sleep Quality in Healthy Adults and Patients with Chronic Conditions: A Systematic Review and Meta-analysis. Journal of Sleep Disorders \& Therapy, 2(141). Retrieved October 10, 2016 from https://www.omicsgroup.org/journals/tai-chi-improves-sleep-quality-in-healthy-adults-andpatients- with-chronic-conditions-a-systematic-review-and-metaanalysis-2167-0277-2141.pdf

Reid, K. J., Baron, K. G., Lu, B., Naylor, E., Wolfe, L., \& Zee, P. C. (2010). Aerobic exercise improves self-reported sleep and quality of life in older adults with insomnia. Sleep Medicine, 11(9), 934-940.

Roth, T. (2007). Insomnia: Definition, prevalence, etiology, and consequences. Journal of Clinical Sleep Medicine, 3(5 suppl), S7-S10.

Rubio-Arias, J. Á., Marín-Cascales, E., Ramos-Campo, D. J., Hernandez, A. V., \& Pérez-López, F. R. (2017). Effect of exercise on sleep quality and insomnia in middle-aged women: A systematic review and meta-analysis of randomized controlled trials, Maturitas, 100, 49-56.

Singh, N. A., Clements, K. M., \& Fiatarone, M. A. (1997) A randomized controlled trial of the effect of exercise on sleep. Sleep, 20(2), 95-101.

Sivertsen, B., Lallukka, T., Salo, P., Pallesen, S., Hysing, M., Krokstad, S., Øverland, S. (2014). Insomnia as a risk factor for ill health: Results from the large population-based prospective HUNT study in Norway. J ournal of Sleep Research, 23(2), 124-132.

Simon, G. E., \& VonKorff, M. (1997). Prevalence, burden, and treatment of insomnia in primary care. The American J ournal of Psychiatry, 154(10), 1417-1423.

Soldatos, C. R., Dikeos, D. G., \& Paparrigopoulos, T. J. (2000). Athens Insomnia Scale: Validation of an instrument based on ICD-10 criteria. Journal of Psychosomatic Research, 48(6), 555-560. 
Stores, G., \& Crawford, C. (1998). Medical student education in sleep and its disorders. Journal of the Royal College Physicians of London, 32(2), 149-153.

Stores, R., \& Wiggs, L. (1998). Sleep education in clinical psychology courses in the UK. Clinical Psychology Forum, 119, 14-18.

Summala, H., \& Mikkola, T. (1994). Fatal accidents among car and truck drivers: effects of fatigue, age, and alcohol consumption. Human Factors, 36(2), 315-326.

Tan, X., Alén, M., Wiklund, P., Partinen, M., \& Cheng, S. (2016). Effects of aerobic exercise on home-based sleep among overweight and obese men with chronic insomnia symptoms: a randomized controlled trial. Sleep Medicine, 25, 113-121.

Tarrier, N., \& Wykes, T. (2004). Is there evidence that cognitive behaviour therapy is an effective treatment for schizophrenia? A cautious or cautionary tale? Behaviour Research and Therapy, 42(12), 1377-401.

Taylor, D. J., Lichstein, K. L., \& Durrence, H. H. (2003) Insomnia as a health risk factor. Behavioural Sleep Medicine, 1(4), 227-247.

Tariq, S. H., \& Pulisetty, S. (2008). Pharmacotherapy for insomnia. Clinics in Geriatric Medicine, 24(1), 93-105.

The National Institute for Health and Care Excellence (NICE, 2004). Guidance on the use of zaleplon, zolpidem and zopiclone for the short-term management of insomnia. Retrieved October 10, 2016 from

https: //www.nice.org.uk/guidance/ta77/chapter/1-Guidance

The PRISMA Group (2009). PRISMA statement. Retrieved August 15, 2016 from http://www. prisma-statement.org/PRISMAStatement/PRISMAStatement. aspx

U.S. Department of Health and Human Service (2008). Physical Activity Guidelines Advisory Committee Report. Retrieved October 10, 2016 from

https://health.gov/paguidelines/report/pdf/CommitteeReport.pdf

van Straten, A., van der Zweerde, T., Kleiboer, A., Cuijpers, P., Morin, C.M., Lancee, J. (2017). Cognitive and behavioral therapies in the treatment of insomnia: A meta-analysis. Sleep Medicine Review. doi: https://doi.org/10.1016/j.smrv.2017.02.001. 
Wang, C., Collet, J.P., \& Lau, J. (2004). The Effect of Tai Chi on Health Outcomes in Patients with Chronic Conditions. A Systematic Review. Archives of Internal Medicine, 164(5), 493-501. doi: $10.1001 /$ archinte. 164.5.493

World Health Organization. (1992). The ICD-10 classification of mental and behavioural disorders. Clinical descriptions and diagnostic guidelines. Geneva: World Health Organization.

World Health Organisation (WHO, 2010). Global Recommendations on Physical Activity for Health. Retrieved September 19, 2016 from http://apps.who.int/iris/bitstream/10665/44399/1/9789241599979_eng.pdf

Wykes, T., Steel, C., Everitt, B., \& Tarrier, N. (2008). Cognitive behavior therapy for schizophrenia: Effect sizes, clinical models, and methodological rigor. Schizophrenia Bulletin, 34(3), 523-537.

Yang, P. Y., Ho, K. H., Chen, H. C., \& Chien, M. Y. (2012). Exercise training improves sleep quality in middle-aged and older adults with sleep problems: a systematic review. Journal of Physiotherapy, 58(3), 157-163.

Youngstedt, S. D. (2005). Effects of exercise on sleep. Clinics in Sports Medicine, 24(2), 355365.

Youngstedt, S. D., O'Connor, P. J., \& Dishman, R. K. (1997). The effects of acute exercise on sleep: a quantitative synthesis. Sleep, 20(3), 203-214.

\begin{tabular}{|l|l|l|l} 
Sample & 1 & Is the sample a convenience sample e.g. -e.g., clinic attenders, referred patients
\end{tabular} 


\begin{tabular}{|c|c|c|}
\hline \multirow[t]{2}{*}{$\begin{array}{l}\text { two } \\
\text { questions } \\
\text { maximum } \\
\text { score }=10\end{array}$} & & $\begin{array}{l}\text { (score } \mathbf{2} \text { ) } \\
\text { OR a geographical cohort e.g. all patients eligible in a particular area (score 5) } \\
\text { OR a highly selective sample e.g. volunteers (score } \mathbf{0} \text { ) }\end{array}$ \\
\hline & 2 & $\begin{array}{l}\text { Is the sample size greater than } 27 \text { participants in each treatment group (score 5) } \\
\text { OR based on described and adequate power calculations (score 5) }\end{array}$ \\
\hline \multirow{3}{*}{$\begin{array}{l}\text { Allocation } \\
\text { three } \\
\text { questions } \\
\text { maximum } \\
\text { score = } 16\end{array}$} & 3 & $\begin{array}{l}\text { Is there true random allocation or minimisation allocation to treatment groups (if } \\
\text { yes score 10) }\end{array}$ \\
\hline & 4 & Is the process of randomisation described (score $\mathbf{3}$ ) \\
\hline & 5 & $\begin{array}{l}\text { Is the process of randomisation carried out independently from the trial research } \\
\text { team (score } \mathbf{3} \text { ) }\end{array}$ \\
\hline \multirow{5}{*}{$\begin{array}{l}\text { Assessment } \\
\text { (for the main } \\
\text { outcome) } \\
\text { five } \\
\text { questions } \\
\text { maximum } \\
\text { score }=32\end{array}$} & 6 & $\begin{array}{l}\text { Are the assessments carried out by independent assessors and not therapists } \\
\text { (score 10) }\end{array}$ \\
\hline & 7 & $\begin{array}{l}\text { Are standardised assessments used to measure symptoms in a standard way } \\
\text { (score 6) } \\
\text { OR idiosyncratic assessments of symptoms (score } \mathbf{3} \text { ) }\end{array}$ \\
\hline & 8 & $\begin{array}{l}\text { Are assessments carried out blind (masked) to treatment group allocation (score } \\
\text { 10) }\end{array}$ \\
\hline & 9 & Are the methods of rater blinding adequately described (score 3) \\
\hline & 10 & Is rater blinding verified (score $\mathbf{3}$ ) \\
\hline $\begin{array}{l}\text { Control } \\
\text { groups } \\
\text { one question } \\
\text { maximum } \\
\text { score = } 16\end{array}$ & 11 & The control group is non-active (if yes score 16) \\
\hline \multirow{2}{*}{$\begin{array}{l}\text { Analysis } \\
\text { two } \\
\text { questions } \\
\text { maximum } \\
\text { score }=15\end{array}$} & 12 & $\begin{array}{l}\text { The analysis is appropriate to the design and the type of outcome measure (score } \\
\text { 5) }\end{array}$ \\
\hline & 13 & $\begin{array}{l}\text { The analysis includes all those participants as randomised (sometimes referred to } \\
\text { as an intention to treat analysis) (score } \mathbf{6} \text { ) } \\
\text { AND an adequate investigation and handling of drop outs from assessment if the } \\
\text { attrition rate exceeds } 15 \% \text { (score } 4 \text { ) }\end{array}$ \\
\hline \multirow{2}{*}{$\begin{array}{l}\text { Active } \\
\text { treatment } \\
\text { three } \\
\text { questions } \\
\text { maximum } \\
\text { score }=11\end{array}$} & 14 & $\begin{array}{l}\text { Was the treatment adequately described (score } \mathbf{3} \text { ) } \\
\text { AND was a treatment protocol or manual used (score 3) }\end{array}$ \\
\hline & 15 & Was adherence to the treatment protocol or treatment quality assessed (score 5) \\
\hline
\end{tabular}

Appendix A: Quality Assessment Tool. 
Appendix B: Quality Ratings of studies included in review.

\begin{tabular}{|c|c|c|c|c|c|c|c|}
\hline Study & $\begin{array}{c}\text { Sample } \\
\text { characteristics }\end{array}$ & $\begin{array}{c}\text { Allocation } \\
\text { to } \\
\text { treatment }\end{array}$ & $\begin{array}{l}\text { Assessment } \\
\text { of outcome }\end{array}$ & $\begin{array}{l}\text { Control } \\
\text { Groups }\end{array}$ & $\begin{array}{c}\text { Description } \\
\text { of } \\
\text { treatments }\end{array}$ & Analysis & $\begin{array}{c}\text { Total } \\
\text { Score } \\
\text { ( Max } \\
=100)\end{array}$ \\
\hline $\begin{array}{l}\text { Afonso et al., } \\
2012\end{array}$ & $0 / 10$ & $10 / 16$ & $16 / 32$ & $16 / 16$ & $8 / 11$ & $5 / 15$ & 55 \\
\hline $\begin{array}{l}\text { Guilleminault } \\
\text { et al., } 1995\end{array}$ & $0 / 10$ & $10 / 16$ & $16 / 32$ & $16 / 16$ & $6 / 11$ & $9 / 15$ & 55 \\
\hline $\begin{array}{l}\text { Hartescu et } \\
\text { al., } 2015\end{array}$ & $5 / 10$ & $16 / 16$ & $32 / 32$ & $16 / 16$ & $8 / 11$ & $15 / 15$ & 92 \\
\hline $\begin{array}{l}\text { Irwin et al., } \\
2014\end{array}$ & $5 / 10$ & $16 / 16$ & $26 / 32$ & $16 / 16$ & $11 / 11$ & $15 / 15$ & 89 \\
\hline $\begin{array}{l}\text { Reid et al., } \\
2010\end{array}$ & $0 / 10$ & $10 / 16$ & $32 / 32$ & $16 / 16$ & $11 / 11$ & $5 / 15$ & 74 \\
\hline $\begin{array}{l}\text { Chan et al., } \\
2016\end{array}$ & $7 / 10$ & $13 / 16$ & $32 / 32$ & $16 / 16$ & $8 / 11$ & $15 / 15$ & 91 \\
\hline $\begin{array}{l}\text { Chen et al., } \\
2016\end{array}$ & $5 / 10$ & $10 / 16$ & $16 / 32$ & $16 / 16$ & $11 / 11$ & $9 / 15$ & 67 \\
\hline $\begin{array}{l}\text { Irwin et al., } \\
2008\end{array}$ & $0 / 10$ & $16 / 16$ & $32 / 32$ & $16 / 16$ & $8 / 11$ & $15 / 15$ & 87 \\
\hline $\begin{array}{l}\text { King et al., } \\
1997\end{array}$ & $5 / 10$ & $13 / 16$ & $32 / 32$ & $16 / 16$ & $8 / 11$ & $15 / 15$ & 89 \\
\hline $\begin{array}{l}\text { King et al., } \\
2008\end{array}$ & $5 / 10$ & $10 / 16$ & $26 / 32$ & $16 / 16$ & $8 / 11$ & $15 / 15$ & 80 \\
\hline $\begin{array}{l}\text { Tan et al., } \\
2016\end{array}$ & $2 / 10$ & $13 / 16$ & $26 / 32$ & $16 / 16$ & $8 / 11$ & $15 / 15$ & 80 \\
\hline
\end{tabular}


SUPPLEMENTARY MATERIAL 
Table S1. Subjective sleep outcomes for insomnia disorder (Raw Data Summaries)

\begin{tabular}{|c|c|c|c|}
\hline Reference & Outcomes & Group (G) & \\
\hline $\begin{array}{l}\text { Afonso et al., } \\
2012\end{array}$ & $\begin{array}{l}\text { ISI } \\
\text { Baseline } \\
\text { Posttreatment }\end{array}$ & $\begin{array}{l}\text { Yoga }(\mathbf{n}=\mathbf{1 5}) \\
14.1(4.65) \\
9.7(4.65)\end{array}$ & $\begin{array}{l}\text { NT }(\mathbf{n}=\mathbf{1 5}) \\
15.2(4.65) \\
13.7(4.65)\end{array}$ \\
\hline $\begin{array}{l}\text { Guilleminault } \\
\text { et al., } 1995\end{array}$ & $\begin{array}{l}\text { Sleep log - TST } \\
\text { Baseline } \\
\text { Posttreatment } \\
\text { Sleep log - SOL } \\
\text { Baseline } \\
\text { Posttreatment }\end{array}$ & $\begin{array}{l}\text { SH+ MAE }(\mathbf{n}=\mathbf{1 0}) \\
299.0(28.0) \\
324.0(34.0) \\
58.0(21.0) \\
47.0(32.0)\end{array}$ & $\begin{array}{l}\text { SH }(\mathbf{n}=10) \\
296.0(39.0) \\
326.0(25.0) \\
44.0(12.0) \\
39.0(17.0)\end{array}$ \\
\hline $\begin{array}{l}\text { Hartescu et al., } \\
2015\end{array}$ & $\begin{array}{l}\text { ISI } \\
\text { Baseline } \\
\text { Posttreatment }\end{array}$ & $\begin{array}{l}\text { MAE }(\mathbf{n}=\mathbf{2 0}) \\
15.82(4.23) \\
11.76(5.88)\end{array}$ & $\begin{array}{l}\text { NT }(\mathbf{n}=\mathbf{2 1}) \\
16.37(5.58) \\
14.95(5.02)\end{array}$ \\
\hline $\begin{array}{l}\text { Irwin et al., } \\
2014\end{array}$ & $\begin{array}{l}\text { PSQI } \\
\text { Baseline } \\
\text { Posttreatment } \\
\text { Follow-up ( } 7 \mathrm{mo}) \\
\text { Follow-up (16mo) } \\
\text { AIS } \\
\text { Baseline } \\
\text { Posttreatment } \\
\text { Follow-up (7mo) } \\
\text { Follow-up (16mo) } \\
\text { PSD - TST } \\
\text { Baseline } \\
\text { Posttreatment } \\
\text { Follow-up (7mo) } \\
\text { Follow-up (16mo) } \\
\text { PSD - SOL } \\
\text { Baseline } \\
\text { Posttreatment } \\
\text { Follow-up (7mo) } \\
\text { Follow-up (16mo) } \\
\text { PSD - SE } \\
\text { Baseline } \\
\text { Posttreatment } \\
\text { Follow-up (7mo) } \\
\text { Follow-up (16mo) } \\
\text { PSD - WASO } \\
\text { Baseline } \\
\text { Posttreatment } \\
\text { Follow-up (7mo) } \\
\text { Follow-up (16mo) }\end{array}$ & $\begin{array}{l}\text { TCC }(\mathbf{n}=\mathbf{4 8}) \\
10.7(3.1) \\
8.0(3.4) \\
7.0(3.9) \\
7.8(3.8) \\
11.7(5.0) \\
7.6(3.0) \\
6.4(3.4) \\
7.0(4.7) \\
327.1(75.54) \\
374.5(76.92) \\
391.5(111.57) \\
389.0(86.63) \\
39.9(34.65) \\
28.4(29.11) \\
25.9(22.87) \\
26.1(27.72) \\
67.5(13.86) \\
75.1(15.94) \\
76.3(20.10) \\
78.0(16.63) \\
64.4(45.74) \\
55.5(54.05) \\
37.8(41.58) \\
36.7(44.35)\end{array}$ & $\begin{array}{l}\text { SS }(\mathbf{n}=\mathbf{2 5}) \\
11.1(2.9) \\
9.6(3.0) \\
8.2(2.6) \\
8.2(3.4) \\
11.6(3.0) \\
9.5(4.0) \\
7.9(3.8) \\
7.6(4.4) \\
342.4(73.5) \\
372.1(124.0) \\
379.0(110.5) \\
381.4(110.5) \\
41.3(24.0) \\
29.1(23.5) \\
27.3(28.0) \\
22.4(16.0) \\
69.6(11.5) \\
74.5(19.0) \\
75.3(19.5) \\
77.7(18.5) \\
80.5(43.0) \\
54.6(40.5) \\
50.2(46.5) \\
51.3(48.0)\end{array}$ \\
\hline $\begin{array}{l}\text { Reid et al., } \\
2010\end{array}$ & $\begin{array}{l}\text { PSQI } \\
\text { Baseline } \\
\text { Posttreatment }\end{array}$ & $\begin{array}{l}\text { SH + MAE }(\mathbf{n}=\mathbf{1 0}) \\
9.9(2.37) \\
5.1(1.44)\end{array}$ & $\begin{array}{l}\text { SH + REA }(\mathbf{n}=\mathbf{7}) \\
8.5(1.76) \\
9.5(1.97)\end{array}$ \\
\hline
\end{tabular}

\section{All values are means (SD).}

Abbreviations AIS - Athens Insomnia Scale; ISI - Insomnia Severity Index; MAE - moderate-intensity aerobic exercise; NT - No treatment; PSD - Pittsburgh Sleep Diary; PSQI - Pittsburgh Sleep Quality Index; REA - 
Recreational and Educational Activities; SH - Sleep Hygiene; SE - Sleep Efficiency; SOL - Sleep Onset Latency; SS - Sleep Seminar; TCC - Tai Chi; TST - Total Sleep Time; WASO - Wake Time After Sleep Onset.

Table S2. Subjective sleep outcomes for insomnia symptoms (Raw Data Summaries)

\begin{tabular}{|c|c|c|c|}
\hline Reference & Outcomes & Group (G) & \\
\hline $\begin{array}{l}\text { Chan et al., } \\
2016\end{array}$ & $\begin{array}{l}\text { PSQI } \\
\text { Baseline } \\
\text { Posttreatment }\end{array}$ & $\begin{array}{l}\text { TCC ( } \mathbf{n}=\mathbf{2 7}) \\
10.2(3.0) \\
7.8(3.3)\end{array}$ & $\begin{array}{l}\text { HE }(\mathbf{n}=\mathbf{2 5}) \\
9.8(3.1) \\
9.6(2.4)\end{array}$ \\
\hline $\begin{array}{l}\text { Irwin et al., } \\
2008\end{array}$ & $\begin{array}{l}\text { PSQI } \\
\text { Baseline } \\
\text { Posttreatment } \\
\text { Follow-up (25wk) }\end{array}$ & $\begin{array}{l}\text { TCC }(\mathbf{n}=30) \\
6.67(1.54) \\
5.13(3.00) \\
4.87(2.30)\end{array}$ & $\begin{array}{l}\text { HE ( } \mathbf{n}=\mathbf{2 2}) \\
8.18(3.25) \\
6.31(3.68) \\
6.97(3.59)\end{array}$ \\
\hline $\begin{array}{l}\text { King et al., } \\
1997\end{array}$ & $\begin{array}{l}\text { PSQI } \\
\text { Baseline } \\
\text { Posttreatment } \\
\text { Sleep diary - SOL } \\
\text { Baseline } \\
\text { Posttreatment }\end{array}$ & $\begin{array}{l}\text { MAE ( } \mathbf{n}=\mathbf{2 4}) \\
8.7(3.0) \\
5.4(2.8) \\
28.4(20.2) \\
14.6(13.0)\end{array}$ & $\begin{array}{l}\text { NT ( } n=24) \\
8.93(3.1) \\
8.8(2.6) \\
26.1(20.0) \\
23.8(15.3)\end{array}$ \\
\hline $\begin{array}{l}\text { King et al., } \\
2008\end{array}$ & $\begin{array}{l}\text { PSQI } \\
\text { Baseline } \\
\text { Posttreatment } \\
\text { Sleep diary - SOL } \\
\text { Baseline } \\
\text { Posttreatment } \\
\text { Sleep diary - TST } \\
\text { Baseline } \\
\text { Posttreatment }\end{array}$ & $\begin{array}{l}\text { MAE ( } \mathbf{n}=\mathbf{3 6}) \\
8.47(3.61) \\
6.36(3.39) \\
38.44(23.32) \\
26.02(18.5) \\
7.53(0.70) \\
7.58(0.78)\end{array}$ & $\begin{array}{l}\text { HE }(\mathbf{n}=30) \\
7.57(2.78) \\
6.57(2.84) \\
23.08(19.01) \\
28.02(21.04) \\
7.42(0.87) \\
7.84(0.80)\end{array}$ \\
\hline $\begin{array}{l}\text { Tan et al., } \\
2016\end{array}$ & $\begin{array}{l}\text { Sleep diary - SOL } \\
\text { Baseline } \\
\text { Posttreatment }\end{array}$ & $\begin{array}{l}\text { PAE ( } n=24) \\
23.9(35.25) \\
13.7(19.88)\end{array}$ & $\begin{array}{l}\text { NT }(\mathbf{n}=\mathbf{2 1}) \\
18.1(24.30) \\
20.7(28.74)\end{array}$ \\
\hline
\end{tabular}

\section{All values are means (SD).}

Abbreviations: HE - Health Education; MAE - Moderate-intensity aerobic exercise; NT - No treatment; PAE Progressive aerobic exercise; PSQI - Pittsburgh Sleep Quality Index; SOL - Sleep Onset Latency; TCC - Tai Chi; TST - Total Sleep Time. 
Table S3. Objective sleep outcomes for insomnia disorder (Raw Data Summaries)

\begin{tabular}{|c|c|c|c|}
\hline Reference & Outcomes & Group (G) & \\
\hline $\begin{array}{l}\text { Afonso et al., } \\
2012\end{array}$ & $\begin{array}{l}\text { PSG - SOL } \\
\text { Baseline } \\
\text { Posttreatment } \\
\text { PSG - TST } \\
\text { Baseline } \\
\text { Posttreatment } \\
\text { PSG - SE } \\
\text { Baseline } \\
\text { Posttreatment } \\
\text { PSG - WASO } \\
\text { Baseline } \\
\text { Posttreatment }\end{array}$ & $\begin{array}{l}\text { Yoga }(\mathbf{n}=\mathbf{1 5}) \\
18.37(18.28) \\
26.45(28.32) \\
351.93(43.03) \\
338.1(42.24) \\
81.95(9.34) \\
78.1(9.13) \\
60.35(30.78) \\
69.51(41.35)\end{array}$ & $\begin{array}{l}\text { NT }(\mathbf{n}=\mathbf{1 5}) \\
16.09(10.70) \\
14.95(20.73) \\
327.83(90.83) \\
351.0(46.53) \\
79.76(6.38) \\
82.31(8.66) \\
74.31(26.78) \\
62.12(31.12)\end{array}$ \\
\hline $\begin{array}{l}\text { Guilleminault } \\
\text { et al., } 1995\end{array}$ & $\begin{array}{l}\text { Actigraphy - SOL } \\
\text { Baseline } \\
\text { Posttreatment } \\
\text { Actigraphy - TST } \\
\text { Baseline } \\
\text { Posttreatment }\end{array}$ & $\begin{array}{l}\text { SH + MAE }(\mathbf{n}=\mathbf{1 0}) \\
33.0(19.0) \\
26.0(20.0) \\
328.0(43.0) \\
345.0(38.0)\end{array}$ & $\begin{array}{l}\text { SH }(\mathbf{n}=\mathbf{1 0}) \\
29.0(13.0) \\
30.0(8.0) \\
338.0(53.0) \\
335.0(29.0)\end{array}$ \\
\hline $\begin{array}{l}\text { Irwin et al., } \\
2014\end{array}$ & $\begin{array}{l}\text { PSG - SOL } \\
\text { Baseline } \\
\text { Posttreatment } \\
\text { PSG - TST } \\
\text { Baseline } \\
\text { Posttreatment } \\
\text { PSG - SE } \\
\text { Baseline } \\
\text { Posttreatment } \\
\text { PSG - WASO } \\
\text { Baseline } \\
\text { Posttreatment }\end{array}$ & $\begin{array}{l}\text { TCC }(\mathbf{n}=\mathbf{4 8}) \\
25.0(24.3) \\
19.0(22.1) \\
378.9(41.2) \\
381.3(54.2) \\
80.1(8.7) \\
80.6(11.2) \\
69.2(37.7) \\
72.5(52.6)\end{array}$ & $\begin{array}{l}\text { SS }(\mathbf{n}=\mathbf{2 5}) \\
33.6(28.5) \\
22.3(22.1) \\
356.4(68.6) \\
378.8(53.7) \\
74.5(14.4) \\
79.5(10.3) \\
88.7(70.7) \\
75.1(43.0)\end{array}$ \\
\hline
\end{tabular}

\section{All values are means (SD).}

Abbreviations: MAE - Moderate-intensity aerobic exercise; NT - No treatment; PSG - polysomnography; SH Sleep hygiene; SE - Sleep Efficiency; SOL - Sleep Onset Latency; SS - Sleep seminar; TCC - Tai Chi; TST Total Sleep Time; WASO - Wake Time After Sleep Onset. 
Table S4. Objective sleep outcomes for insomnia symptoms (Raw Data Summaries)

\begin{tabular}{|c|c|c|c|}
\hline Reference & Outcomes & Group (G) & \\
\hline $\begin{array}{l}\text { Chen et al., } \\
2016\end{array}$ & $\begin{array}{l}\text { Actigraphy - SOL } \\
\text { Baseline } \\
\text { Posttreatment } \\
\text { Actigraphy - TST } \\
\text { Baseline } \\
\text { Posttreatment } \\
\text { Actigraphy - SE } \\
\text { Baseline } \\
\text { Posttreatment } \\
\text { Actigraphy - WASO } \\
\text { Baseline } \\
\text { Posttreatment }\end{array}$ & $\begin{array}{l}\text { MAE ( } \mathbf{n}=\mathbf{2 9}) \\
13.8(7.55) \\
12.5(3.77) \\
330.8(58.21) \\
342.9(63.60) \\
75.5(7.55) \\
82.2(5.93) \\
83.0(31.26) \\
76.5(35.04)\end{array}$ & $\begin{array}{l}\text { NT }(\mathbf{n}=\mathbf{3 4 )} \\
17.4(10.49) \\
20.4(11.08) \\
333.8(52.47) \\
344.5(50.14) \\
75.0(7.58) \\
76.3(7.58) \\
84.2(41.98) \\
91.4(40.23)\end{array}$ \\
\hline $\begin{array}{l}\text { King et al., } \\
2008\end{array}$ & $\begin{array}{l}\text { PSG - SOL } \\
\text { Baseline } \\
\text { Posttreatment } \\
\text { PSG - TST } \\
\text { Baseline } \\
\text { Posttreatment } \\
\text { PSG - SE } \\
\text { Baseline } \\
\text { Posttreatment }\end{array}$ & $\begin{array}{l}\text { MAE ( } \mathbf{n}=\mathbf{3 6}) \\
15.06(17.12) \\
14.22(12.23) \\
361.58(60.34) \\
361.09(65.48) \\
80.95(9.52) \\
79.89(10.53)\end{array}$ & $\begin{array}{l}\text { HE (n = 30) } \\
17.74(11.68) \\
18.42(13.55) \\
361.94(63.58) \\
373.95(54.64) \\
82.46(9.37) \\
81.09(10.36)\end{array}$ \\
\hline $\begin{array}{l}\text { Tan et al., } \\
2016\end{array}$ & $\begin{array}{l}\text { Sleep Monitoring System } \\
\text { SOL } \\
\text { Baseline } \\
\text { Posttreatment } \\
\text { TST } \\
\text { Baseline } \\
\text { Posttreatment } \\
\text { SE } \\
\text { Baseline } \\
\text { Posttreatment } \\
\text { WASO } \\
\text { Baseline } \\
\text { Posttreatment }\end{array}$ & $\begin{array}{l}\text { PAE }(\mathbf{n}=\mathbf{2 4}) \\
23.9(35.25) \\
13.7(19.88) \\
376.8(57.63) \\
390.1(63.13) \\
81.7(10.5) \\
87.1(7.75) \\
48.3(22.88) \\
39.1(20.50)\end{array}$ & $\begin{array}{l}\text { NT }(\mathbf{n}=\mathbf{2 1}) \\
18.1(24.30) \\
20.7(28.74) \\
393.3(52.46) \\
412.8(52.34) \\
85.9(7.36) \\
86.6(7.48) \\
44.8(14.25) \\
38.7(17.41)\end{array}$ \\
\hline
\end{tabular}

\section{All values are means (SD).}

Abbreviations: HE - Health education; MAE - Moderate-intensity aerobic exercise; NT - No treatment; PAE Progressive aerobic exercise; PSG - polysomnography; SE - sleep efficiency; SOL - sleep onset latency; TST Total Sleep Time; WASO - Wake Time After Sleep Onset. 